\title{
Moduli of spherical varieties and log geometry
}

\author{
Martin Olsson
}

\begin{abstract}
We give moduli interpretations using log geometry of the main components of the moduli spaces of polarized spherical varieties defined by Alexeev and Brion. The key new insight is that logarithmic structures alone do not suffice to give a good moduli interpretation. Instead we introduce the notion of an enriched log structure, which is a logarithmic structure together with some extra data related to the group action on the varieties being parametrized.
\end{abstract}

\section{Introduction}

This paper is a continuation of our earlier work [Ols08a, Ols08b] on isolating main components of moduli spaces using log geometry in the sense of Fontaine, Illusie, and Kato. In this paper we extend our logarithmic interpretation of the main component in Alexeev's moduli spaces for broken toric varieties, studied in [Ols08a], to the main components in the moduli spaces for stable spherical varieties introduced by Alexeev and Brion in [AB06].

The basic moduli problem considered in [AB06] can loosely be described as follows. Throughout we work over an algebraically closed field $k$ of characteristic 0 . Let $G / k$ be a connected reductive algebraic group over $k$ and fix a representation $V$ of $G$. Alexeev and Brion then consider moduli of pairs $(X, f)$, where $X$ is a projective variety with action of $G$ and

$$
f: X \longrightarrow \mathbb{P} V
$$

is a $G$-equivariant finite morphism satisfying some conditions (it is a stable spherical variety with weight set $\Gamma$; see $[\mathrm{AB} 06, \S 4])$. In $[\mathrm{AB} 06,4.2]$ it is shown that this moduli problem is representable by a proper algebraic stack with finite diagonal $\mathscr{M}_{\Gamma, \mathbb{P} V}$.

Fix a pair $\left(X_{0}, f_{0}\right)$ over $k$ with $X_{0}$ irreducible, and let $L_{0}$ denote the ample invertible sheaf $f_{0}^{*} \mathscr{O}_{\mathbb{P} V}(1)$. For suitably chosen $\left(X_{0}, L_{0}\right)$, there is an open substack $\mathscr{M}_{\Gamma, \mathbb{P} V,\left(X_{0}, L_{0}\right)}^{\circ} \subset \mathscr{M}_{\Gamma, \mathbb{P} V}$ classifying pairs $(X, f)$ such that the corresponding polarized scheme with $G$-action $\left(X, f^{*} \mathscr{O}_{\mathbb{P} V}(1)\right)$ is isomorphic to $\left(X_{0}, L_{0}\right)$. We let $\mathscr{M}_{\Gamma, \mathbb{P} V,\left(X_{0}, L_{0}\right)} \subset \mathscr{M}_{\Gamma, \mathbb{P} V}$ denote the scheme-theoretic closure of $\mathscr{M}_{\Gamma, \mathbb{P} V,\left(X_{0}, L_{0}\right)}^{\circ}$.

An analysis of 1-parameter degenerations exhibits toric phenomena very similar to the case of toric varieties and polarized abelian varieties studied in [Ols08a], and this suggests there may be a moduli interpretation using log geometry of $\mathscr{M}_{\Gamma, \mathbb{P} V,\left(X_{0}, L_{0}\right)}$. The aim of this paper is to explain such a moduli interpretation.

Received 4 June 2014, accepted in final form 26 February 2015.

2010 Mathematics Subject Classification 14D22, 14L30.

Keywords: moduli, spherical variety, main component, log geometry.

This journal is (C) Foundation Compositio Mathematica 2015. This article is distributed with Open Access under the terms of the Creative Commons Attribution Non-Commercial License, which permits non-commercial reuse, distribution, and reproduction in any medium, provided that the original work is properly cited. For commercial re-use, please contact the Foundation Compositio Mathematica.

The author was partially supported by NSF CAREER grant DMS-0748718 and NSF grant DMS-1303173. 


\section{OLSSON}

A degenerating family $\mathcal{X}$ of stable spherical varieties (say over a trait $S$ ) does have a natural $\log$ structure $M_{\mathcal{X}}$, but the induced morphism of $\log$ schemes $\left(\mathcal{X}, M_{\mathcal{X}}\right) \rightarrow\left(S, M_{S}\right)$, where $M_{S}$ denotes the standard $\log$ structure on $S$, is not $\log$ smooth. Perhaps more significantly, the deformation theory of the pair $\left(\mathcal{X}, M_{\mathcal{X}}\right)$ is not well behaved and one does not get a good moduli stack using just log structures. To remedy this situation we introduce the new notion of an enrichment on the $\log$ structure $M_{\mathcal{X}}$. The definition is technical but the basic idea can be understood as follows (for full details see Section 8).

Fix a Borel subgroup $B$ of $G$, a maximal torus $T \subset B \subset G$, let $U \subset B$ be the unipotent radical of $B$, and let $\left(X_{0}, L_{0}\right)$ be an irreducible polarized spherical variety (in the setting of $(X, f)$ as above, the line bundle is $\left.f^{*} \mathscr{O}_{\mathbb{P} V}(1)\right)$. One can then consider the ring with $\widetilde{G}:=G \times \mathbb{G}_{m}$-action $A:=\oplus_{n \geqslant 0} \Gamma\left(X_{0}, L_{0}^{\otimes n}\right)$ and the corresponding cone $C_{X_{0}}:=\operatorname{Spec}(A)$, an affine spherical variety in the sense of $[\mathrm{AB} 06,1.3]$. The ring of $U$-invariants $A^{U}$ with its natural $T$-action then defines a toric variety. Roughly speaking, the log structure alluded to above only sees information about $A^{U}$ and its degenerations, but this is not enough to understand the entire ring $A$. Our notion of an enriched log structure is a way to understand the degeneration of the multiplicative structure on $A$ and not just on $A^{U}$.

More precisely, let $\Lambda$ be the character group of $T$ and let $\Lambda^{+} \subset \Lambda$ be the dominant weights. Then the character group of $\widetilde{T}:=T \times \mathbb{G}_{m} \subset \widetilde{G}$ is $\Lambda \times \mathbb{Z}$ and the dominant weights $\widetilde{\Lambda}^{+} \subset \widetilde{\Lambda}$ are given by $\Lambda^{+} \times \mathbb{Z}$. Let $P \subset \widetilde{\Lambda}^{+}$be the dominant weights of the irreducible representations which occur in $A$. We then have

$$
A=\oplus_{p \in P} A_{p},
$$

where $A_{p}$ is a representative for the irreducible representation corresponding to $p$. To understand the component of the moduli space of polarized spherical varieties containing $\left(X_{0}, L_{0}\right)$ we essentially have to understand how to deform the multiplicative structure on $A$. For each $p \in P$ we have a line $L_{p} \subset A_{p}$ of highest weight vectors, and

$$
A^{U}=\oplus_{p \in P} L_{p} .
$$

Using the toric structure we can therefore find an isomorphism $A^{U} \simeq k[P]$ compatible with the torus actions, or equivalently highest weight vectors $v_{p} \in A_{p}$ such that $v_{p} \cdot v_{q}=v_{p+q}$ (multiplication in $A$ ). For each $p, q, r$ with $A_{r}$ occurring in $A_{p} \cdot A_{q}$ let

$$
m_{p, q}^{r}: A_{p} \otimes A_{q} \longrightarrow A_{r}
$$

be the $r$ th component of the multiplication map. To get families of spherical varieties over a ring $R$ we can then change the multiplicative structure by multiplying the $m_{p, q}^{r}$ by elements $\eta_{p, q}^{r} \in R^{*}$ (or if we want to consider degenerations, we take $\eta_{p, q}^{r} \in R$ ). These elements have to satisfy a number of compatibilities to make the resulting $R$-module $A \otimes_{k} R$ an algebra. Note that the variation in algebra structure on $A^{U}$ obtained in this way is determined by the elements $\eta_{p, q}^{p+q}$, but to work with the whole algebra $A$ we need also the elements $\eta_{p, q}^{r}$.

To globalize this discussion we consider logarithmic structures. We consider proper integral morphisms of log schemes $\left(f, f^{b}\right):\left(X, M_{X}\right) \rightarrow\left(T, M_{T}\right)$ together with a relatively ample line bundle $L$ on $X$ and an isomorphism $\alpha: P \simeq f_{*} M_{X / T}$, where $M_{X / T}$ is the cokernel in the category of sheaves of integral monoids of the map $f^{b}: f^{*} M_{T} \rightarrow M_{X}$. This data is required to satisfy a number of conditions, among them that the map $f_{*} M_{X} \rightarrow f_{*} M_{X / T}$ be surjective. For each $p \in P$ the set of liftings $L_{p}$ (the notation here is consistent with the preceding paragraph; $L_{p}$ is a global version of the torsor of highest weight vectors) of $\alpha(p)$ to $f_{*} M_{X}$ has a natural action of $M_{T}$ and in fact is what we call an invertible $M_{T}$-set (see Section 2). These invertible $M_{T}$-sets behave in 


\section{MODULI OF SPHERICAL VARIETIES AND LOG GEOMETRY}

many ways like torsors, and in particular for two invertible $M_{T}$-sets $L$ and $L^{\prime}$ we can form a third $L \wedge L^{\prime}$, and more generally for an invertible $M_{T}$-set $L$ and an arbitrary $M_{T}$-set $N$ we can form $L \wedge N$. A logarithmic enrichment is a collection of maps

$$
\eta_{p, q}^{r}: L_{p} \wedge L_{q} \longrightarrow M_{T} \wedge L_{r}
$$

for each $p, q, r \in P$ with $A_{r}$ occurring in $A_{p} \cdot A_{q}$. These maps $\eta_{p, q}^{r}$ are required to satisfy a number of natural compatibilities (see Definition 8.6).

In the case when $G$ is a torus, the enrichment on the log structure is no additional information and our theory reduces to the logarithmic interpretation of the main components of moduli spaces for broken toric varieties studied in [Ols08a].

Using the notion of enriched log structures, we relate the main components $\mathscr{M}_{\Gamma, \mathbb{P} V,\left(X_{0}, L_{0}\right)}$ to a suitable moduli problem. The main result is Theorem 10.6. We construct a moduli problem classifying enriched polarized log schemes with $G$-action and show that it is representable by a proper algebraic stack $\mathscr{M}_{\Gamma, \mathbb{P} V,\left(X_{0}, L_{0}\right)}^{\log }$. Furthermore, there is a natural morphism

$$
\tau^{\prime}: \mathscr{M}_{\Gamma, \mathbb{P} V,\left(X_{0}, L_{0}\right)}^{\log } \longrightarrow \mathscr{M}_{\Gamma, \mathbb{P} V,\left(X_{0}, L_{0}\right)}
$$

which is a universal homeomorphism.

\section{Conventions}

All sheaves of monoids are assumed commutative with unit and integral. From Section 3 onward we work over an algebraically closed field $k$ of characteristic 0 .

\section{Background: invertible $M$-sets}

2.1. Let $C$ be a site and $M$ a sheaf of monoids on $C$.

Following [Ogu14, Definition 1.4.5], an invertible $M$-set is a sheaf of sets $S$ with an action of $M$ such that for every object $U \in C$ there exist a covering $\left\{U_{i} \rightarrow U\right\}_{i \in I}$ and sections $u_{i} \in S\left(U_{i}\right)$ such that the maps $\left.\left.M\right|_{C / U_{i}} \rightarrow S\right|_{C / U_{i}}$ induced by $m \mapsto m \cdot u_{i}$ are isomorphisms. An isomorphism of invertible $M$-sets $S \rightarrow S^{\prime}$ is an isomorphism of sheaves of sets compatible with the $M$-action.

Note that if $M$ is a sheaf of groups, then the notion of invertible $M$-set is equivalent to the notion of $M$-torsor.

If $f: M \rightarrow N$ is a morphism of sheaves of monoids and $S$ is an invertible $M$-set, then we can form the pushout $N \wedge^{M} S$ by taking the quotient of $N \times S$ by the equivalence relation generated by $(f(m)+n, s) \sim(n, m \cdot s)$ for local sections $n \in N, m \in M$, and $s \in S$. The action of $N$ induced by the action on the first factor makes $N \wedge^{M} S$ an invertible $N$-set.

In particular, if $M^{*} \subset M$ denotes the subsheaf of invertible elements, then there is a functor

$$
\left.\left(M^{*} \text {-torsors }\right) \longrightarrow \text { (invertible } M \text {-sets }\right) \text {. }
$$

Proposition 2.2 (See also the discussion following [Ogu14, Definition 1.4.5]). The functor (2.1.1) is an equivalence of categories.

Proof. Both sides of (2.1.1) form stacks over $C$ and every object of the right side is locally in the image by definition. So it suffices to show that the functor is fully faithful, which is immediate.

2.3. Invertible $M$-sets will arise for us in the following way. Let $\left(X, M_{X}\right)$ be a fine $\log$ scheme, and let $M_{X} \rightarrow N$ be an injective integral morphism of sheaves of integral monoids on $X$. Assume 


\section{OLSSON}

that the quotient $N / M_{X}$ is isomorphic to a constant sheaf $P$, and that for every specialization of geometric points $\bar{\eta} \rightarrow \bar{x}$ the induced diagram

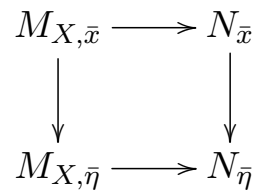

is cocartesian. Let $\pi: N \rightarrow P$ be the projection, and for $p \in P$ let $S_{p} \subset N$ be the sheaf $\pi^{-1}(p)$.

Proposition 2.4. The natural action of $M_{X}$ on $S_{p}$ makes $S_{p}$ an invertible $M_{X}$-set.

Proof. Let $\bar{x} \rightarrow X$ be a geometric point. Let us first show that the $M_{X, \bar{x}}$-set $S_{p, \bar{x}}$ is invertible. Indeed, by [Ogu14, Proposition 2.1.5] the set $S_{p, \bar{x}}$ contains a minimal element $q \in S_{p, \bar{x}}$. Let $t \in S_{p, \bar{x}}$ be another element. Then since $t$ and $q$ have the same image in $P$, there exist elements $m_{1}, m_{2} \in M_{X, \bar{x}}$ such that $t+m_{1}=q+m_{2}$. Since the map $M_{X, \bar{x}} \rightarrow N_{\bar{x}}$ is integral, we can find an element $t_{0} \in N_{\bar{x}}$ and elements $m_{3}, m_{4} \in M_{X, \bar{x}}$ such that $t=t_{0}+m_{3}, q=t_{0}+m_{4}$, and $m_{1}+m_{3}=m_{2}+m_{4}$. Since $q$ is a minimal element, the element $m_{4}$ must be a unit, whence $t=q+\left(m_{3}-m_{4}\right)$.

Fix a geometric point $\bar{x} \rightarrow X$ and let $U \rightarrow X$ be an étale neighborhood of $\bar{x}$ over which there exists a section $s \in S_{p}(U)$ whose image $s_{\bar{x}}$ in $S_{p, \bar{x}}$ generates $S_{p, \bar{x}}$. We claim that after possibly further shrinking on $U$ the section $s$ defines an isomorphism $M_{X} \rightarrow S_{p}$. Let $\bar{S}_{p}$ denote the quotient $S_{p} / \mathscr{O}_{X}^{*}$, so $\bar{S}_{p}$ is a $\bar{M}_{X}$-set. A section $s$ of $S_{p}$ generates $S_{p}$ if and only if its image in $\bar{S}_{p}$ generates $\bar{S}_{p}$. Since the sheaf $\bar{S}_{p}$ is constructible, it therefore suffices to show that the set of points $\bar{\eta} \rightarrow U$ for which the section $\bar{s} \in \bar{S}_{p}$ induced by $s$ generates $\bar{S}_{p, \bar{\eta}}$ is stable under generization.

To prove this let $\bar{\eta} \rightarrow U$ be a geometric point specializing to a point $\bar{y} \rightarrow U$ for which $\bar{s}_{y} \in \bar{S}_{p, \bar{y}}$ is a generator. By our assumptions the map $\bar{S}_{p, \bar{y}} \rightarrow \bar{S}_{p, \bar{\eta}}$ is surjective. Let $\rho \in \bar{S}_{p, \bar{\eta}}$ be a minimal element and let $\tilde{\rho} \in \bar{S}_{p, \bar{y}}$ be a lifting of $\rho$. Since $\bar{s}_{p, \bar{y}}$ is minimal, there exists an element $m \in \bar{M}_{X, \bar{y}}$ such that $\tilde{\rho}=\bar{s}_{p, \bar{y}}+m$. Therefore $\bar{s}_{p, \bar{\eta}}$ is also in the sub- $\bar{M}_{X, \bar{\eta}}$-set of $S_{p, \bar{\eta}}$ generated by $\bar{s}_{p, \bar{\eta}}$, whence $\bar{s}_{p, \bar{\eta}}$ generates $\bar{S}_{p, \bar{\eta}}$.

2.5. For $p \in P$ let $L_{p}$ denote the $\mathscr{O}_{X}^{*}$-torsor corresponding to $S_{p}$ under the equivalence (2.1.1). The sheaf $L_{p}$ is the subsheaf of $S_{p}$ of sections which generate $S_{p}$. For two sections $e_{p} \in L_{p}$ and $e_{q} \in L_{q}$ their sum $e_{p}+e_{q}$ inside $N$ is an element of $S_{p+q}$. We can therefore write $e_{p}+e_{q}$ as $m+e_{p+q}$ for some elements $m \in M_{X}$ and $e_{p+q} \in L_{p+q}$. The pair $\left(m, e_{p+q}\right)$ is not unique, but any other such pair is of the form $\left(m+u, e_{p+q}-u\right)$ for some element $u \in \mathscr{O}_{X}^{*}$. We therefore get a well-defined element of the quotient $M_{X} \wedge^{\mathscr{O}_{X}^{*}} L_{p+q}$ of the product $M_{X} \times L_{p+q}$ by the action of $\mathscr{O}_{X}^{*}$ given by $u *(m, e)=(m+u, e-u)$. In this way we obtain a map of $\mathscr{O}_{X}^{*}$-sets

$$
\theta_{p, q}: L_{p} \wedge^{\mathscr{O}_{X}^{*}} L_{q} \longrightarrow M_{X} \wedge^{\mathfrak{O}_{X}^{*}} L_{p+q}
$$

\section{Spherical varieties: the basics}

In this paper we follow the conventions and notation of [AB06]. For the convenience of the reader and to fix the notation we review some of the notation and definitions from loc. cit.

3.1. For the remainder of the paper we fix the following notation. Let $k$ be an algebraically closed field of characteristic 0 . Fix a connected reductive group $G / k$, and let $B \subset G$ be a Borel 


\section{MODULI OF SPHERICAL VARIETIES AND LOG GEOMETRY}

subgroup with maximal torus $T \subset B$ and unipotent radical $U$. We write $\Lambda$ for the character group of $T$ and $\Lambda^{+} \subset \Lambda$ for the dominant weights.

If $V$ is a finite-dimensional irreducible $G$-representation, then there exists a unique $B$-invariant line $L \subset V$ on which $B$ acts through a character $\chi \in \Lambda^{+}$. This establishes a bijection between the set of isomorphism classes of irreducible representations of $G$ and $\Lambda^{+}$. We fix for each $\lambda \in \Lambda^{+}$ a corresponding irreducible representation $V(\lambda)$.

Definition 3.2. (i) A G-variety over $k$ is a connected, reduced, separated, finite type $k$-scheme $X$ with an action of $G$ over $k$.

(ii) A polarized $G$-variety over $k$ is a pair $(X, L)$ consisting of a projective $G$-variety $X$ and an ample $G$-linearized invertible sheaf $L$.

(iii) An affine $G$-variety $X$ over $k$ is multiplicity free if the multiplicity of any irreducible representation occurring in $\Gamma\left(X, \mathscr{O}_{X}\right)$ is at most 1 .

(iv) A polarized $G$-variety $(X, L)$ is multiplicity free if for every $n$ and irreducible representation $V$ of $G$ the multiplicity of $V$ in the representation $\Gamma\left(X, L^{\otimes n}\right)$ is at most 1 .

3.3. Let $\widetilde{G}$ denote the group $\mathbb{G}_{m} \times G$, which has Borel subgroup $\widetilde{B}:=\mathbb{G}_{m} \times B$ and maximal torus $\widetilde{T}:=\mathbb{G}_{m} \times T$. Let $\widetilde{\Lambda}=\mathbb{Z} \times \Lambda$ be the corresponding character group and let $\widetilde{\Lambda}^{+}=\mathbb{Z} \times \Lambda^{+}$be the dominant weights. If $(X, L)$ is a polarized $G$-variety, then we can form the $\widetilde{G}$-representation

$$
R(X, L):=\oplus_{n \geqslant 0} \Gamma\left(X, L^{\otimes n}\right)
$$

and the $\widetilde{G}$-variety $\widetilde{X}:=\operatorname{Spec}(R(X, L))$. The scheme $\widetilde{X}$ is called the cone of $(X, L)$.

An affine irreducible $G$-variety $X$ is spherical if it is normal and multiplicity free. A polarized $G$-variety $(X, L)$ is spherical if $X$ is normal and $(X, L)$ is multiplicity free.

3.4. For an affine $G$-variety $X$ define the weight set $\Lambda^{+}(X) \subset \Lambda^{+}$to be the set of $\lambda \in \Lambda^{+}$for which $V(\lambda)$ occurs in the representation $\Gamma\left(X, \mathscr{O}_{X}\right)$, and the weight cone $C(X)$ to be the cone $\mathbb{R}_{\geqslant 0} \Lambda^{+}(X) \subset \Lambda_{\mathbb{R}}$ generated by $\Lambda^{+}(X)$.

For a polarized $G$-variety define the weight set $\widetilde{\Lambda}^{+}(X, L) \subset \widetilde{\Lambda}^{+}$to be $\Lambda^{+}(\widetilde{X})$, and the weight cone $\widetilde{C}(X, L) \subset \widetilde{\Lambda}_{\mathbb{R}}$ to be the cone $C(\widetilde{X})$. Also define the moment set $Q(X, L)$ to be

$$
Q(X, L):=\widetilde{C}(X, L) \cap\left(\{1\} \times \Lambda_{\mathbb{R}}^{+}\right) \subset \widetilde{\Lambda}_{\mathbb{R}}=\mathbb{R} \times \Lambda_{\mathbb{R}} .
$$

Then $\widetilde{C}(X, L)$ is the cone over $Q(X, L)$. If $X$ is irreducible, then $Q(X, L)$ is a rational convex polytope in $\Lambda_{\mathbb{R}}^{+}$called the moment polytope.

3.5. Fix a subgroup $\Gamma \subset \Lambda$. A stable affine spherical variety with weights in $\Gamma$ is an affine $G$-variety $X$ satisfying the following:

(i) The variety $X$ is seminormal.

(ii) The variety $X$ contains only finitely many $G$-orbits and they are spherical.

(iii) For any $G$-orbit closure $Y$ the weight group $\Lambda(Y)$ (the subgroup of $\Lambda$ generated by the weight set of $Y$ ) is contained in $\Gamma$ as a direct summand.

Similarly, for a subgroup $\Gamma \subset \widetilde{\Lambda}$ a polarized stable spherical variety with weights in $\Gamma$ is a polarized $G$-variety $(X, L)$ satisfying the following:

(i) The variety $X$ is seminormal. 


\section{OLSSON}

(ii) The variety $X$ contains only finitely many $G$-orbits and they are spherical.

(iii) For any $G$-orbit closure $Y$ the weight group $\widetilde{\Lambda}(Y, L)$, which is defined to be the subgroup of $\widetilde{\Lambda}$ generated by $\widetilde{\Lambda}^{+}(Y, L)$, is contained in $\Gamma$ as a direct summand.

3.6. These definitions extend to families as follows. For a scheme $S / k$ and subgroup $\Gamma \subset \Lambda$ a stable affine spherical variety over $S$ with weights in $\Gamma$ is an $S$-scheme $f: X \rightarrow S$ with action of $G$ such that the following hold:

(i) The morphism $f$ is flat and locally of finite presentation.

(ii) For every $\lambda \in \widetilde{\Lambda}^{+}$, with corresponding representation $V(\lambda)$, the sheaf $\mathscr{H}_{o m_{G}}\left(V(\lambda), f_{*} \mathscr{O}_{X}\right)$ is a locally free sheaf of rank 0 or 1 .

(iii) Every geometric fiber of $f$ is a stable affine spherical variety with weights in $\Gamma$.

Similarly, for a subgroup $\Gamma \subset \widetilde{\Lambda}$ a polarized stable spherical variety over $S$ with weights in $\Gamma$ is a pair $(f: X \rightarrow S, L)$, such that the following hold:

(i) The morphism $f$ is proper, flat, and locally of finite presentation and $G$ acts on $X$ over $S$.

(ii) The sheaf $L$ is a relatively ample invertible sheaf on $X$.

(iii) Every geometric fiber of $f$ is a polarized stable spherical variety with weights in $\Gamma$.

If $(X / S, L)$ is a polarized stable spherical variety over a connected scheme $S$ with weights in $\Gamma$, then the weight sets of all geometric fibers are the same.

\section{Pavings}

4.1. With notation as in Section 3, fix a subgroup $\Gamma \subset \widetilde{\Lambda}$ and a polytope $Q \subset \Gamma_{\mathbb{R}}$, and let $(X, L)$ be a polarized stable spherical variety with moment set $Q$ and weights in $\Gamma$.

Let $Y \subset X$ be the closure of a $G$-orbit in $X$. Then by [AB06, Proposition 2.1.3] the pair $\left(Y,\left.L\right|_{Y}\right)$ is again a spherical variety, and by [AB06, Proposition 2.2.3(iii)] the restriction map

$$
H^{0}\left(X, L^{\otimes n}\right) \longrightarrow H^{0}\left(Y,\left.L^{\otimes n}\right|_{Y}\right)
$$

is surjective for all $n$. This implies that if $R$ (respectively, $R_{Y}$ ) denotes the coordinate ring of the cone $\widetilde{X}$ (respectively, $\widetilde{Y}$ ) then the induced map $R \rightarrow R_{Y}$ is surjective. Let $Q_{Y} \subset Q$ be the moment polytope of $\left(Y,\left.L\right|_{Y}\right)$. Then it follows from [AB06, Lemma 2.3.1] that the set of subpolytopes $S=\left\{Q_{Y}\right\}$ obtained from the closures $Y$ of $G$-orbits in $X$ form a paving of $Q$ in the sense of [Ols08a, 3.1.1].

4.2. Pavings that arise from closed fibers of 1-parameter degenerations of irreducible spherical varieties can be described as follows. Let $V$ be a complete discrete valuation ring over $k$ with field of fractions $K$, residue field $F$, and uniformizer $\pi \in \mathfrak{m}_{V}$. Let $(\mathscr{X}, \mathscr{L}) / V$ be a stable spherical variety over $V$ with irreducible generic fiber and with moment polytope $Q$. Let $\mathcal{R}$ be the coordinate ring of $\widetilde{\mathscr{X}}$, and let $\mathcal{R}_{\eta}$ (respectively, $\mathcal{R}_{s}$ ) denote the coordinate ring of the generic (respectively, closed) fiber of $\widetilde{\mathscr{X}}$. We have a $G$-equivariant inclusion $\mathcal{R} \hookrightarrow \mathcal{R}_{\eta}$.

By [AB06, Proposition 3.1.2] we can, after replacing $V$ by a finite extension, find a stable spherical variety $\left(X_{0}, L_{0}\right)$ over $k$ such that $\left(\mathscr{X}_{\eta}, \mathscr{L}_{\eta}\right)$ is isomorphic to $\left(X_{0}, L_{0}\right) \otimes_{k} K$. We fix one such isomorphism. Let $R_{0}$ be the coordinate ring of the cone of $\left(X_{0}, L_{0}\right)$, so $\mathcal{R}_{\eta} \simeq R_{0} \otimes_{k} K$. For $\lambda$ in the weight set of $(X, L)$ with corresponding representation $V(\lambda)$ let $R_{0, \lambda} \subset R_{0}$ (respectively, 


\section{MODULI OF SPHERICAL VARIETIES AND LOG GEOMETRY}

$\left.\mathcal{R}_{\lambda} \subset \mathcal{R}\right)$ denote the image of the natural map

$$
V(\lambda) \otimes \operatorname{Hom}_{\widetilde{G}}\left(V(\lambda), R_{0}\right) \longrightarrow R_{0} \quad\left(\text { respectively, } V(\lambda) \otimes \operatorname{Hom}_{\widetilde{G}}(V(\lambda), \mathcal{R}) \longrightarrow \mathcal{R}\right) .
$$

Then there exists a unique integer $h(\lambda)$ for which

$$
\mathcal{R}_{\lambda}=\pi^{h(\lambda)} R_{0, \lambda} \otimes_{k} V
$$

as submodules of $\mathcal{R}_{\eta}$.

Let $P \subset \Gamma$ denote the intersection $\widetilde{C}(X, L) \cap \Gamma$, and let $P_{Y} \subset \Gamma$ denote $\widetilde{C}\left(Y,\left.L\right|_{Y}\right) \cap \Gamma$. By [AB06, Proposition 1.3.1(v)] the weight set of $(X, L)$ (respectively, $\left(Y,\left.L\right|_{Y}\right)$ ) is equal to $P$ (respectively, $P_{Y}$ ).

Lemma 4.3. The function $h$ is linear on each $P_{Y}$.

Proof. Let $\lambda, \eta \in P_{Y}$ be two elements and let $v_{\lambda}$ (respectively, $v_{\eta}, v_{\lambda+\eta}$ ) be a highest weight vector in $R_{0, \lambda}$ (respectively, $R_{0, \eta}, R_{0, \lambda+\eta}$ ). Then the product

$$
\left(\pi^{h(\lambda)} v_{\lambda}\right) \cdot\left(\pi^{h(\eta)} v_{\eta}\right)
$$

has $(\eta+\lambda)$-component given by

$$
\pi^{h(\lambda)+h(\eta)-h(\lambda+\eta)} \cdot\left(\pi^{h(\lambda+\eta)}\right) \cdot v_{\lambda+\eta} .
$$

Since this product reduces to something nonzero modulo $\pi$, this implies

$$
h(\lambda)+h(\eta)=h(\lambda+\eta) .
$$

4.4. In particular, we get a function $\bar{h}$ on the rational points of $Q$ by the formula

$$
\bar{h}(p / \operatorname{deg}(p))=\frac{1}{\operatorname{deg}(p)} h(p) .
$$

LEMma 4.5. The function $\bar{h}$ is piecewise linear on the rational points of $Q$, convex, with domains of linearity precisely the polytopes $\left\{Q_{Y}\right\}$.

Proof. We have already seen that $\bar{h}$ is linear on each of the polytopes $Q_{Y}$. Furthermore, for two elements $\lambda, \eta \in P$ we must have

$$
h(\lambda)+h(\eta)-h(\lambda+\eta) \geqslant 0,
$$

since for highest weight vectors $v_{\lambda}$ and $v_{\eta}$ as above we must have

$$
\left(\pi^{h(\lambda)} v_{\lambda}\right) \cdot\left(\pi^{h(\eta)} \cdot v_{\eta}\right) \subset V \cdot \pi^{h(\lambda+\eta)} v_{\lambda+\eta} .
$$

It remains to see that the domains of linearity are precisely the $Q_{Y}$. For this let $\lambda, \eta \in P$ be two elements which do not lie in a common $Q_{Y}$, and let $v_{\lambda} \in R_{0, \lambda}$ and $v_{\eta} \in R_{0, \eta}$ be highest weight vectors. Then the product

$$
\left(\pi^{h(\lambda)} v_{\lambda}\right) \cdot\left(\pi^{h(\eta)} v_{\eta}\right) \in \mathcal{R}
$$

maps to zero in the coordinate ring of each irreducible component of the closed fiber. It follows that this product lies in $\pi^{h(\lambda+\eta)+1} V \cdot R_{0, \lambda+\eta}$, whence $h(\lambda)+h(\eta)>h(\lambda+\eta)$. This implies that the polytopes $\left\{Q_{Y}\right\}$ are exactly the domains of linearity of the function $\bar{h}$.

DeFinition 4.6. Let $\left(X_{0}, L_{0}\right)$ be an irreducible polarized spherical variety over $k$. 


\section{OLSSON}

(i) A piecewise linear function $\bar{h}: Q \rightarrow \mathbb{R}$ is admissible with respect to $\left(X_{0}, L_{0}\right)$ if there exists a family of polarized spherical varieties $(\mathscr{X}, \mathscr{L})$ over a discrete valuation ring $V$ such that $\left(\mathscr{X}_{\eta}, \mathscr{L}_{\eta}\right) \simeq\left(X_{0}, L_{0}\right) \otimes_{k} \operatorname{Frac}(V)$, such that $\bar{h}$ is the function obtained by the above construction.

(ii) A paving of $Q$ is regular with respect to $\left(X_{0}, L_{0}\right)$ if the polytopes of the paving are precisely the domains of linearity of an admissible function with respect to $\left(X_{0}, L_{0}\right)$.

Remark 4.7. In Lemma 5.2 below we give a more combinatorial characterization of the condition that a piecewise linear function be admissible with respect to $\left(X_{0}, L_{0}\right)$.

\section{Encoding multiplication maps}

5.1. Let $K$ be a field of characteristic 0 (there is no harm in considering just $K=k$, but later we will also want to consider extensions of $k$ ) and fix an irreducible polarized spherical $G$-variety $(X, L)$ over $K$. Let $R$ denote the ring $R(X, L)$, and let $P$ denote the weight set $\widetilde{\Lambda}(X, L)$. Let $Q$ denote the moment polytope of $(X, L)$. As in $\S 4.2$, for $a \in P$ let $R_{a} \subset R$ denote the image of $\operatorname{Hom}_{\widetilde{G}}(V(a), R) \otimes V(a) \rightarrow R$, so $R_{a}$ is noncanonically isomorphic to $V(a)$ as $\widetilde{G}$-representations. For $a, b, c \in P$ let

$$
m_{a, b}^{c}: R_{a} \otimes R_{b} \longrightarrow R_{c}
$$

denote the composite of the multiplication map $R_{a} \otimes R_{b} \rightarrow R$ with the projection onto $R_{c}$.

For $a, b, c \in P$ write $(a, b) \succ c$ (or $c \prec(a, b))$ if $R_{c}$ occurs with nonzero multiplicity in $R_{a} \cdot R_{b} \subset R$.

Lemma 5.2. A piecewise linear function $\bar{h}: Q \rightarrow \mathbb{R}$ is admissible with respect to $(X, L)$ if and only if the corresponding function $h: P \rightarrow \mathbb{R}$ defined by $h(a):=\operatorname{deg}(a) \cdot \bar{h}(a / \operatorname{deg}(a))$ has values in the natural numbers $\mathbb{N}$ and for every $a, b, c \in P$ with $(a, b) \succ c$ we have

$$
h(a)+h(b)-h(c) \geqslant 0 .
$$

Proof. It follows from the construction that an admissible function has the indicated properties. Conversely, let $\mathcal{R} \subset R \otimes_{K} K((t))$ denote the $K[[t]]$-submodule given by $\oplus_{a \in P} t^{h(a)} K[[t]] \otimes R_{a}$. The assumptions on $h$ imply that $\mathcal{R}$ is in fact a subalgebra of $R \otimes_{K} K((t))$. It follows from [AB06, Lemma 2.1.5] that $\mathcal{R}$ defines a stable spherical variety over $K[[t]]$, whence $\bar{h}$ is admissible with respect to $(X, L)$.

5.3. Let $F$ be the free abelian group with generators $\eta_{a, b}^{c}$, one for each triple $a, b, c \in P$ with $(a, b) \succ c$, and let $\widetilde{E}$ be the quotient of $F$ by the following relations:

(i) For all $a, b \in P$ we have $\eta_{a, b}^{a+b}=0$.

(ii) For all $(a, b) \succ c$ we have $\eta_{a, b}^{c}=\eta_{b, a}^{c}$.

(iii) For all $a, b, c, d, e, f \in P$ (some of them possibly equal) with

$$
e \prec(a, b), d \prec(e, c), f \prec(b, c) \text {, and } d \prec(a, f)
$$

we have

$$
\eta_{e, c}^{d}+\eta_{a, b}^{e}=\eta_{a, f}^{d}+\eta_{b, c}^{f} .
$$

(iv) For all $a, b, c, d, e, f \in P$ with

$$
e \prec(a, b), d \prec(e, c), f \prec(a, b) \text {, and } d \prec(f, c)
$$




\section{MODULI OF SPHERICAL VARIETIES AND LOG GEOMETRY}

we have

$$
\eta_{a, b}^{f}+\eta_{c, f}^{d}=\eta_{a, b}^{e}+\eta_{c, e}^{d}
$$

There is a map

$$
\tilde{\epsilon}: \widetilde{E} \longrightarrow P^{g p}
$$

sending $\eta_{a, b}^{c}$ to $a+b-c$.

Proposition 5.4. Let $E$ be the quotient of $\widetilde{E}$ by its torsion subgroup. Then the map $\epsilon: E \rightarrow P^{\text {gp }}$ induced by $\tilde{\epsilon}$ is injective.

Proof. Note that the formation of $\widetilde{E}, E, P$, etc. is invariant under field extensions of $K$. For the proof we may therefore without loss of generality assume that $K$ is algebraically closed.

Consider the group algebra $K[E]$, and let $S$ denote $\operatorname{Spec}(K[E])$. Define a graded $K[E]$-algebra with $\widetilde{G}$-action $\mathcal{R}$ as follows. The underlying graded module with $\widetilde{G}$-action is just $R \otimes_{K} K[E]$. The multiplication map, however, is given by summing the maps

$$
\eta_{a, b}^{c} \cdot m_{a, b}^{c}:\left(R_{a} \otimes K[E]\right) \otimes_{K[E]}\left(R_{b} \otimes K[E]\right) \longrightarrow R_{c} \otimes K[E]
$$

over all triples $a, b, c$ with $(a, b) \succ c$.

Lemma 5.5. This definition gives $\mathcal{R}$ the structure of a commutative $K[E]$-algebra.

Proof. The only nontrivial point is that this multiplication map is associative. To ease notation, for $a \in P$ write $\mathcal{V}_{a}$ for $R_{a} \otimes K[E]$ and let

$$
\chi_{a, b}^{c}: \mathcal{V}_{a} \otimes \mathcal{V}_{b} \longrightarrow \mathcal{V}_{c}
$$

be the $c$ th factor of the multiplication map, so we have $\chi_{a, b}^{c}=\eta_{a, b}^{c} \cdot m_{a, b}^{c}$ if $c \prec(a, b)$ and $\chi_{a, b}^{c}=0$ otherwise. The associativity condition amounts to the statement that for $a, b, c, d \in P$ we have

$$
\sum_{e \prec(a, b), d \prec(c, e)} \chi_{e, c}^{d} \circ\left(\chi_{a, b}^{e} \otimes \mathrm{id}\right)=\sum_{f \prec(b, c), d \prec(a, f)} \chi_{a, f}^{d} \circ\left(\mathrm{id} \otimes \chi_{b, c}^{f}\right) .
$$

Now observe that relations (iii) and (iv) in the definition of $\widetilde{E}$ imply that there exists a single element $\eta_{a, b, c}^{d} \in E$ such that

$$
\sum_{e \prec(a, b), d \prec(c, e)} \chi_{e, c}^{d} \circ\left(\chi_{a, b}^{e} \otimes \mathrm{id}\right)=\eta_{a, b, c}^{d} \cdot \sum_{e \prec(a, b), d \prec(c, e)} m_{e, c}^{d} \circ\left(m_{a, b}^{e} \otimes \mathrm{id}\right)
$$

and

$$
\sum_{f \prec(b, c), d \prec(a, f)} \chi_{a, f}^{d} \circ\left(\mathrm{id} \otimes \chi_{b, c}^{f}\right)=\eta_{a, b, c}^{d} . \sum_{f \prec(b, c), d \prec(a, f)} m_{a, f}^{d} \circ\left(\mathrm{id} \otimes m_{b, c}^{f}\right) .
$$

The associativity then follows from the fact that $R$ is an associative algebra.

The spectrum of $R^{U}$, where as in $\S 3.1$ we let $U$ denote the unipotent radical of $B$, is an affine toric variety with torus $D\left(P^{\mathrm{gp}}\right)$, and therefore is isomorphic to $\operatorname{Spec}(K[P])$. Fix one such isomorphism. Then by construction we have an induced isomorphism $\mathcal{R}^{U} \simeq \operatorname{Spec}(K[E \rtimes P])$, where $E \rtimes P$ is defined as in [Ols08a, 3.1.9].

Let $\Omega$ denote the field of fractions of $K[E]$, and consider the $\widetilde{G}$-variety $\operatorname{Spec}\left(\mathcal{R}_{\Omega}\right)$. It comes equipped with an isomorphism $\sigma: \mathcal{R}_{\Omega}^{U} \simeq \Omega[P]$ (since $\eta_{a, b}^{a+b}=0$ for all $a, b \in P$ ). 


\section{OLSSON}

Lemma 5.6. There exist an affine $\widetilde{G}$-variety $\operatorname{Spec}\left(R^{\prime}\right)$ over $K$ such that $R^{\prime U}$ is isomorphic to $K[P]$ and a finite extension $\Omega^{\prime}$ of $\Omega$ over which we have an isomorphism of $\widetilde{G}$-varieties $\delta: \operatorname{Spec}\left(R^{\prime} \otimes_{K}\right.$ $\left.\Omega^{\prime}\right) \simeq \operatorname{Spec}\left(\mathcal{R}_{\Omega} \otimes_{\Omega} \Omega^{\prime}\right)$.

Proof. Let

$$
M_{K[P]}:(K \text {-schemes })^{\text {op }} \longrightarrow \text { Set }
$$

be the functor, defined in [AB05, Definition 1.11], sending a $K$-scheme $S$ to the set of equivalence classes of pairs $(X, \iota)$, where $X$ is an affine $\widetilde{G}$-scheme over $S$ and $\iota: X / / U \rightarrow \operatorname{Spec}(K[P]) \times_{\operatorname{Spec}(K)}$ $S$ is a $T$-equivariant isomorphism (see loc. cit. for more details). There is an action of the torus $D\left(P^{\mathrm{gp}}\right)$ on $M_{K[P]}$ : for a scheme $S$ an element $\alpha \in D\left(P^{\mathrm{gp}}\right)(S)$ sends an element $[(X, \iota)] \in$ $M_{K[P]}(S)$ to $[(X, \alpha \circ \iota)] \in M_{K[P]}$, where we write also $\alpha$ for the corresponding automorphism of $\operatorname{Spec}(K[P]) \times_{\operatorname{Spec}(K)} S$. By [AB05, Theorem 1.12] the functor $M_{K[P]}$ is representable by a quasi-projective $K$-scheme, and by [AB05, Corollary 3.4] the action of $D\left(P^{\mathrm{gp}}\right)$ on $M_{K[P]}$ has only finitely many orbits.

The pair $\left(\operatorname{Spec}\left(\mathcal{R}_{\Omega}\right), \sigma\right)$ then defines a point of $M_{K[P]}(\Omega)$. Since the action of $D\left(P^{\mathrm{gp}}\right)$ on $M_{K[P]}$ has only finitely many orbits, we can find a finite extension $\Omega^{\prime}$ of $\Omega$ over which there exists an element $\alpha \in D\left(P^{\mathrm{gp}}\right)\left(\Omega^{\prime}\right)$ such that $\left(\operatorname{Spec}\left(\mathcal{R}_{\Omega^{\prime}}\right), \alpha \circ \sigma\right)$ defines a $K$-point of $M_{K[P]}$. The corresponding $\widetilde{G}$-variety $\operatorname{Spec}\left(R^{\prime}\right)$ over $K$ then has the desired properties.

With notation as in Lemma 5.6 fix also an isomorphism of toric varieties $\lambda$ : $\operatorname{Spec}\left(R^{\prime U}\right) \simeq$ $\operatorname{Spec}(K[P])$. The isomorphism $\delta$ induces by taking $U$-invariants an isomorphism of toric varieties

$$
\operatorname{Spec}\left(\Omega^{\prime}[P]\right) \stackrel{\sigma}{\longrightarrow} \operatorname{Spec}\left(\mathcal{R}_{\Omega^{\prime}}^{U}\right) \stackrel{\delta^{-1}}{\longrightarrow} \operatorname{Spec}\left(R^{\prime U} \otimes_{K} \Omega^{\prime}\right) \stackrel{\lambda}{\longrightarrow} \operatorname{Spec}\left(\Omega^{\prime}[P]\right),
$$

which corresponds to an element $u \in D\left(P^{\mathrm{gp}}\right)\left(\Omega^{\prime}\right)$.

With notation as in Lemma 5.6 observe that the natural map $\iota: E \rightarrow \Omega$ induced by the standard map $E \rightarrow K[E]^{*}$ is injective and that $E \cap K^{*}=\{1\}$. We can read off this map $\iota$ from $\mathcal{R}_{\Omega}$ and the given identifications $\sigma_{a}:\left(\mathcal{R}_{\Omega}\right)_{a} \simeq R_{a} \otimes_{K} \Omega$ using the multiplicative structure on $\mathcal{R}_{\Omega}$ (5.4.1). Similarly, we get a map $\iota^{\prime}: E \rightarrow K$ from the pair $\left(\operatorname{Spec}\left(R^{\prime}\right), \lambda\right)$. The existence of the isomorphism $\delta$ implies that the composition of $\iota^{\prime}$ with the inclusion $K \hookrightarrow \Omega^{\prime}$ is equal to the map

$$
E \longrightarrow \Omega^{\prime}, \quad e \longmapsto u(e) \cdot \iota(e) \text {. }
$$

In particular, $\iota$ and $\iota^{\prime}$ agree on the kernel $\kappa$ of the map $E \rightarrow P^{\mathrm{gp}}$, so $\iota(\kappa) \subset E \cap K^{*}=0$. It follows that $\kappa=0$.

\section{The standard construction}

In this section we construct certain families of degenerating spherical varieties with log structures. The construction is a generalization of the toric case [Ols08a, 3.1].

6.1. Fix an irreducible polarized spherical $G$-variety $(X, L)$ over $k$, which we will refer to as the starting variety, and let $\widetilde{X}$ be its cone, an affine $\widetilde{G}$-variety. Let $Q=Q(X, L) \subset \Lambda_{\mathbb{R}}^{+}$be the corresponding rational convex polytope. Let $R$ be the affine coordinate ring of $\widetilde{X}$, so we have a decomposition

$$
R \simeq \oplus_{\lambda \in \widetilde{\Lambda}^{+}} R_{\lambda}
$$

where each $R_{\lambda}$ equals $F_{\lambda} \otimes V(\lambda)$ for a $k$-vector space $F_{\lambda}$ of dimension 0 or 1 . 


\section{MODULI OF SPHERICAL VARIETIES AND LOG GEOMETRY}

Let $S$ be a paving of $Q$ regular with respect to $(X, L)$.

6.2. For $\omega \in S$ let $P_{\omega}$ denote the intersection $P \cap \operatorname{Cone}(\omega)$. For $\eta \subset \omega$ we have a natural inclusion $P_{\eta} \subset P_{\omega}$. Let

$$
P_{S}:=\underset{\omega \in S}{\lim _{\omega}} P_{\omega}
$$

be the colimit in the category of integral monoids, and let $P_{S}^{\mathrm{gp}}$ be its associated group. By the universal property of the group associated to a monoid we have

$$
P_{S}^{\mathrm{gp}}=\underset{\omega \in S}{\lim _{\omega}} P_{\omega}^{\mathrm{gp}}
$$

For $\omega \in S$ let $\rho_{\omega}: P_{\omega} \rightarrow P_{S}^{\mathrm{gp}}$ be the natural map, and define $\rho: P \rightarrow P_{S}^{\mathrm{gp}}$ to be the set map sending $p \in P_{\omega}$ to $\rho_{\omega}(p)$ (as in [Ols08a, 3.1.4] the map $\rho$ is well defined). Define $K_{S} \subset P_{S}^{\mathrm{gp}}$ to be the submonoid generated by elements of the form

$$
\rho(\lambda)+\rho(\mu)-\rho(\eta)
$$

where $\lambda, \mu, \eta \in P$ are such that $(\lambda, \mu) \succ \eta$.

Remark 6.3. We will see below that the monoid $K_{S}$ is finitely generated, but this does not follow immediately from the definition.

Remark 6.4. In [Ols08a, 3.1.4] we considered the submonoid $H_{S} \subset K_{S}$ generated by elements of the form $\rho(\lambda)+\rho(\mu)-\rho(\lambda+\mu)$. In the case when $G$ is a torus we have $H_{S}=K_{S}$, but in general $K_{S}$ is bigger than $H_{S}$.

Remark 6.5. There is a map $P_{S}^{\mathrm{gp}} \rightarrow P^{\mathrm{gp}}$ induced by the inclusions $P_{\omega}^{\mathrm{gp}} \hookrightarrow P^{\mathrm{gp}}$. In [Los09], the image of an element $\rho(\lambda)+\rho(\mu)-\rho(\eta)$ in $P^{\text {gp }}$ is called a tail of $\widetilde{X}$, and the real cone generated by the image of $K_{S}$ in $\widetilde{\Lambda}_{\mathbb{R}}$ is called the tail cone.

6.6. Let $\mathcal{R}$ denote the $k\left[K_{S}\right]$-module $R \otimes_{k} k\left[K_{S}\right]$, and consider the map

$$
\mathcal{R} \otimes_{k\left[K_{S}\right]} \mathcal{R} \longrightarrow \mathcal{R}
$$

sending $f \otimes g \in R_{\lambda} \otimes R_{\mu}$ to the element whose $\eta$-component is given by the $\eta$-component of $f g$ (multiplication in $R$ ) multiplied by the element $\rho(\lambda)+\rho(\mu)-\rho(\eta) \in K_{S}$.

Lemma 6.7. (i) This gives $\mathcal{R}$ the structure of a commutative $k\left[K_{S}\right]$-algebra.

(ii) There exist a finite-dimensional $k$-representation $V$ of $G$ and a $G$-equivariant surjection

$$
k\left[K_{S}\right] \otimes \operatorname{Sym}^{\prime}(V) \longrightarrow \mathcal{R} .
$$

In particular, $\mathcal{R}$ is a $k\left[K_{S}\right]$-algebra of finite type.

Proof. For part (i) it suffices to show that for every homomorphism $g: k\left[K_{S}\right] \rightarrow A$ to a discrete valuation ring sending all elements of $K_{S}$ to nonzero elements of $A$ we get an algebra structure on $\mathcal{R} \otimes_{k\left[K_{S}\right]} A$. Fix a uniformizer $\pi \in A$. The homomorphism $g$ corresponds to two morphisms of monoids $u: K_{S} \rightarrow A^{*}$ and $h: K_{S} \rightarrow \mathbb{N}$ defined by the formula

$$
g(m)=u(m) \cdot \pi^{h(m)}, \quad m \in K_{S} .
$$

After replacing $A$ by a finite extension (which is harmless for the purposes of verifying that $\mathcal{R}$ is an algebra) we can extend these morphisms to morphisms

$$
\tilde{u}: \underset{\omega}{\lim _{\omega}} P_{\omega}^{\mathrm{gp}} \longrightarrow A^{*}, \quad \tilde{h}: \underset{\omega}{\lim } P_{\omega}^{\mathrm{gp}} \longrightarrow \mathbb{Z} .
$$




\section{OLSSON}

We then get an injective morphism of $A$-modules with $G$-action

$$
\mathcal{R} \otimes_{k\left[K_{S}\right]} A \longrightarrow R \otimes_{k} \operatorname{Frac}(A),
$$

which on $R_{\lambda} \otimes_{k} A \subset \mathcal{R} \otimes_{k\left[K_{S}\right]} A$ is given by $\tilde{u}(\lambda) \pi^{\tilde{h}(\lambda)}$ times the standard inclusion $R_{\lambda} \otimes_{k} A \hookrightarrow$ $R_{\lambda} \otimes_{k} \operatorname{Frac}(A)$. This inclusion is compatible with the multiplication operations, from which it follows that $\mathcal{R} \otimes_{k\left[K_{S}\right]} A$ is a commutative $A$-algebra.

To prove part (ii) consider first a single $\omega \in S$ and choose a set of generators $\lambda_{1}, \ldots, \lambda_{r} \in P_{\omega}$. Choosing isomorphisms $R_{\lambda_{i}} \simeq V\left(\lambda_{i}\right)$, we obtain a morphism

$$
k\left[K_{S}\right] \otimes_{k} \operatorname{Sym}\left(V\left(\lambda_{1}\right) \oplus \cdots \oplus V\left(\lambda_{r}\right)\right) \longrightarrow \mathcal{R} .
$$

If $v_{\lambda_{i}} \in V\left(\lambda_{i}\right)$ denotes a highest weight vector, then the image of $v_{\lambda_{1}}^{\otimes n_{1}} \otimes \cdots \otimes v_{\lambda_{r}}^{\otimes n_{r}}$ (for integers $\left.n_{i} \geqslant 0\right)$ maps to a highest weight vector in $k\left[K_{S}\right] \otimes_{k} V\left(n_{1} \lambda_{1}+\cdots+n_{r} \lambda_{r}\right) \subset \mathcal{R}$. It follows that the image of (6.7.2) contains $\mathcal{R}_{\lambda}$ for all $\lambda \in P_{\omega}$. Repeating the above construction for each $\omega$, we then find a finite-dimensional representation $V$ of $G$ and a surjective map (6.7.1) as desired.

6.8. A more subtle issue is to show that the algebra $\mathcal{R}$ is finitely presented as a $k\left[K_{S}\right]$-algebra. Since $k\left[K_{S}\right]$ is not a priori noetherian, this is not immediate from the above. In fact, we will show that $\mathcal{R}$ is indeed finitely presented and then deduce from this that $K_{S}$ is a finitely generated monoid.

Fix a $G$-representation $V$ and a surjective map (6.7.1) as in Lemma 6.7(ii). Let $\mathbb{A}(V)$ denote $\operatorname{Spec}(\operatorname{Sym}(V))$, and let $h: \widetilde{\Lambda}^{+} \rightarrow \mathbb{N}$ be the function sending all elements of the weight set $\widetilde{\Lambda}^{+}(X, L)$ to 1 and other elements to 0 . We can then consider the equivariant Hilbert functor $\operatorname{Hilb}_{h}^{\widetilde{G}}(\mathbb{A}(V))$ defined in [AB05, Definition 1.5], which assigns to a $k$-scheme $W$ the set of finitely presented $\widetilde{G}$-equivariant closed subschemes $Z \hookrightarrow \mathbb{A}(V)_{W}$ flat over $T$ and with Hilbert function $h$. By $\left[\mathrm{AB} 05\right.$, Theorem 1.7] the functor $\operatorname{Hilb}_{h}^{\widetilde{G}}(\mathbb{A}(V))$ is a quasi-projective scheme.

Let $\mathbb{A}(V) / / U$ denote the spectrum of $\operatorname{Sym}^{\cdot}(V)_{\lambda}^{U}$, an affine scheme with action of the torus $\widetilde{T}$. We can then also consider the $\widetilde{T}$-equivariant Hilbert scheme $\operatorname{Hilb}_{h} \widetilde{T}(\mathbb{A}(V) / / U)$. By $[\mathrm{AB} 05$, Theorem 1.7] there is a closed embedding

$$
q: \operatorname{Hilb}_{h}^{\widetilde{G}}(\mathbb{A}(V)) \longleftrightarrow \operatorname{Hilb}_{h}^{\widetilde{T}}(\mathbb{A}(V) / / U)
$$

sending $Z \hookrightarrow \mathbb{A}(V)_{W}$ to $Z / / U \hookrightarrow(\mathbb{A}(V) / / U)_{W}$.

The restriction of $\mathcal{R}$ to $k\left[K_{S}^{\mathrm{gp}}\right]$ is finitely presented as $k\left[K_{S}^{\mathrm{gp}}\right]$ is noetherian. The surjection (6.7.1) restricted to $k\left[K_{S}^{\mathrm{gp}}\right]$ therefore defines a morphism

$$
b: \operatorname{Spec}\left(k\left[K_{S}^{\mathrm{gp}}\right]\right) \longrightarrow \operatorname{Hilb}_{h}^{\widetilde{G}}(\mathbb{A}(V)) .
$$

Lemma 6.9. The morphism $b$ extends to a morphism $b^{\prime}: \operatorname{Spec}\left(k\left[K_{S}\right]\right) \rightarrow \operatorname{Hilb}_{h}^{\widetilde{G}}(\mathbb{A}(V))$.

Proof. Fix an isomorphism $R^{U} \simeq k[P]$ compatible with the $\widetilde{T}$-actions. Let $H_{S} \subset K_{S}$ be the submonoid defined in [Ols08a, 3.1.4]. Then with notation as in [Ols08a, 3.1.9] we have

$$
\mathcal{R}^{U} \simeq k\left[P \rtimes H_{S}\right] \otimes_{k\left[H_{S}\right]} k\left[K_{S}\right] .
$$

By [Ols08a, Corollary 3.1.8] the monoid $H_{S}$ is finitely generated, from which it follows that $\mathcal{R}^{U}$ is finitely presented over $k\left[K_{S}\right]$. The algebra $\mathcal{R}^{U}$ therefore defines a morphism

$$
a: \operatorname{Spec}\left(k\left[K_{S}\right]\right) \longrightarrow \operatorname{Hilb}_{h}^{\widetilde{T}}(\mathbb{A}(V) / / U)
$$




\section{MODULI OF SPHERICAL VARIETIES AND LOG GEOMETRY}

fitting into a commutative diagram of solid arrows

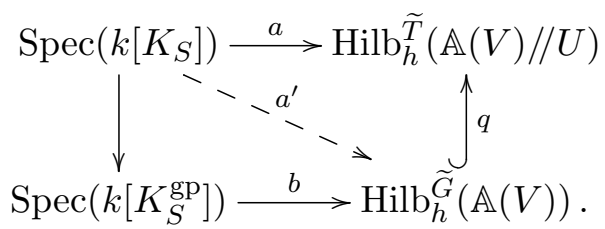

Since $q$ is a closed embedding, it follows that the indicated morphism $a^{\prime}$ exists.

Corollary 6.10. The $k\left[K_{S}\right]$-algebra $\mathcal{R}$ is finitely presented.

Proof. The morphism $a^{\prime}$ corresponds to a surjection

$$
k\left[K_{S}\right] \otimes \operatorname{Sym}^{\prime}(V) \longrightarrow \mathcal{R}^{\prime}
$$

which, since $q \circ a^{\prime}=a$, gives a factorization of (6.7.1) as

$$
k\left[K_{S}\right] \otimes \operatorname{Sym} \cdot(V) \longrightarrow \mathcal{R}^{\prime} \longrightarrow \mathcal{R}
$$

with $\mathcal{R}^{\prime} \rightarrow \mathcal{R}$ an isomorphism over $k\left[K_{S}^{\mathrm{gp}}\right]$. Since $\mathcal{R}^{\prime}$ and $\mathcal{R}$ are both flat over $k\left[K_{S}\right]$, this implies $\mathcal{R}^{\prime} \simeq \mathcal{R}$.

Corollary 6.11. The monoid $K_{S}$ is finitely generated.

Proof. Since $\mathcal{R}$ is finitely presented over $k\left[K_{S}\right]$, there exist a finitely generated submonoid $M \subset$ $K_{S}$ such that $M^{\mathrm{gp}}=K_{S}^{\mathrm{gp}}$ and a model $\mathcal{R}_{0}$ over $k[M]$ with an isomorphism $\mathcal{R}_{0} \otimes_{k[M]} k\left[K_{S}\right] \simeq \mathcal{R}$. This gives an embedding of $k[M]$-algebras with $\widetilde{G}$-action $\mathcal{R}_{0} \hookrightarrow R \otimes_{k} k\left[K_{S}^{\text {gp }}\right]$. Now, if $V(\eta)$ occurs in $R_{\lambda} \cdot R_{\mu}$, then the $\eta$-component of $\mathcal{R}_{0, \lambda} \cdot \mathcal{R}_{0, \mu}$ must be contained in $R_{\eta} \otimes k[M]$, which implies $K_{S} \subset M$, whence $M=K_{S}$.

6.12. There is a $\log$ structure on $\operatorname{Spec}(\mathcal{R})$ defined as follows. The ring of invariants $R^{U}$ is a toric variety with torus $D\left(P^{\text {gp }}\right)$. Choose an isomorphism $R^{U} \simeq k[P]$. If $e^{p} \in R^{U}$ is the "monomial" corresponding to $p \in P$, then the image of $e^{p}$ in $R_{\lambda}$ is a highest weight vector $v_{\lambda} \in R_{\lambda}$ (which is noncanonically isomorphic to $V(\lambda)$ ). These highest weight vectors $v_{\lambda}$ have the property that $v_{\lambda} \cdot v_{\mu}$ equals $v_{\lambda+\mu}$ in $R$.

Let $K_{S} \rtimes P$ be the monoid whose underlying set is the product $K_{S} \times P$ but with additive structure given by

$$
(h, p)+\left(h^{\prime}, p^{\prime}\right):=\left(\left(\rho(p)+\rho\left(p^{\prime}\right)-\rho\left(p+p^{\prime}\right)\right)+h+h^{\prime}, p+p^{\prime}\right) .
$$

See [Ols08a, 3.1.9] for a further discussion of this construction. We then get a morphism of monoids

$$
K_{S} \rtimes P \longrightarrow \mathcal{R}
$$

induced by the set map $P \rightarrow \mathcal{R}$ sending $p$ to $v_{p}$ and the natural map $K_{S} \rightarrow k\left[K_{S}\right]$. This defines a log structure $M_{\mathcal{R}}$ on $\operatorname{Spec}(\mathcal{R})$. Furthermore, if $M_{K_{S}}$ denotes the log structure on $\operatorname{Spec}\left(k\left[K_{S}\right]\right)$ defined by $K_{S} \rightarrow k\left[K_{S}\right]$, then we get a morphism of fine log schemes

$$
\left(\tilde{f}, \tilde{f}^{b}\right):\left(\operatorname{Spec}(\mathcal{R}), M_{\mathcal{R}}\right) \longrightarrow\left(\operatorname{Spec}\left(k\left[K_{S}\right]\right), M_{K_{S}}\right) .
$$

There is an action of $\widetilde{B}$ on $\left(\operatorname{Spec}(\mathcal{R}), M_{\mathcal{R}}\right)$ over $\left(\operatorname{Spec}\left(k\left[K_{S}\right]\right), M_{K_{S}}\right)$. In particular, removing the origin in $\operatorname{Spec}(\mathcal{R})$ and taking the quotient by $\mathbb{G}_{m}$, we obtain a family of polarized log schemes with $B$-action

$$
\left(f, f^{b}\right):\left(\mathscr{X}, M_{\mathscr{X}}, L_{\mathscr{X}}\right) \longrightarrow\left(\operatorname{Spec}\left(k\left[K_{S}\right]\right), M_{K_{S}}\right),
$$




\section{OLSSON}

as well as an extension of the $B$-action to a $G$-action on the underlying scheme $\mathscr{X}$.

Lemma 6.13. Let deg: $P \rightarrow \mathbb{N}$ be the map corresponding to the grading on $P$. Then for every $n \geqslant 0$ the natural map

$$
\oplus_{\lambda \in P, \operatorname{deg}(\lambda)=n} \mathcal{R}_{\lambda} \longrightarrow f_{*} L_{\mathscr{X}}^{\otimes n}
$$

is an isomorphism, and the formation of $f_{*} L_{\mathscr{X}}^{\otimes n}$ commutes with arbitrary base change $S \rightarrow$ $\operatorname{Spec}\left(k\left[K_{S}\right]\right)$.

Proof. This follows from the same argument as in [AB06, Proposition 2.2.3 and discussion following Definition 3.2.1].

6.14. Let $\mathscr{C}^{\circ}$ denote the complement of the origin in $\operatorname{Spec}(\mathcal{R})$, and let $M_{\mathscr{C}} \circ$ denote the pullback of the $\log$ structure $M_{\mathscr{X}}$ to $\mathscr{C}^{\circ}$. The chart $K_{S} \rtimes P \rightarrow M_{\mathcal{R}}$ on $\operatorname{Spec}(\mathcal{R})$ induces a map $K_{S} \rtimes P \rightarrow$ $\bar{M}_{\mathscr{C}}$ o which is $\widetilde{B}$-equivariant, and hence descends to a morphism $\gamma: K_{S} \rtimes P \rightarrow \bar{M}_{\mathscr{X}}$, which locally lifts to a chart.

6.15. There is a morphism $k\left[K_{S}\right] \rightarrow k$ defined as follows. Let $K_{S}^{\text {sat, } \times} \subset K_{S}^{\text {sat }}$ denote the units in the saturation of $K_{S}$. Then the quotient $\bar{K}_{S}^{\mathrm{sat}, \mathrm{gp}}:=K_{S}^{\mathrm{gp}} / K_{S}^{\mathrm{sat}, \times}$ is torsion free, and therefore there exists a (noncanonical) splitting $K_{S}^{\text {sat }} \simeq K_{S}^{\text {sat, } \times} \oplus \bar{K}_{S}^{\text {sat }}$. This implies that there exists a unique homomorphism $K_{S}^{\text {sat }} \rightarrow k$ (viewed as a multiplicative monoid) sending $K_{S}^{\text {sat, } \times}$ to 1 and the nonunits of $K_{S}^{\text {sat }}$ to 0 . Let $v: k\left[K_{S}\right] \rightarrow k$ denote the induced morphism, and let $k\left[\left[K_{S}\right]\right]$ denote the completion of $k\left[K_{S}\right]$ with respect to this maximal ideal.

6.16. Let $\beta: K_{S} \rightarrow k$ be an arbitrary morphism. Define a new paving $S^{\prime}$ of $Q$ which is coarser than $S$ as follows. By blowing up $k\left[K_{S}\right]$ along $\beta$ and looking at a generic point of the exceptional fiber, we can find a field extension $L$ of $k$ and an inclusion $\tilde{\beta}$ : $\left[K_{S}\right] \hookrightarrow A$ into a discrete valuation ring $A$ with residue field $L$ such that the diagram

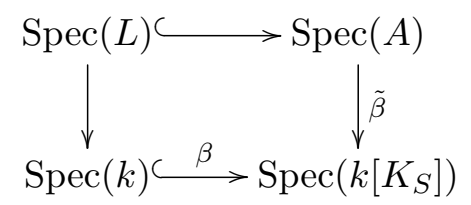

commutes. Let $\rho: K_{S} \rightarrow \mathbb{N}$ be the map induced by the map $\tilde{\beta}$ and the valuation on $A$, and write also $\rho: K_{S}^{\mathrm{gp}} \rightarrow \mathbb{Z}$ for the induced map on groups. Let $\mathcal{R}_{A}$ denote the base change of $\mathcal{R}$ to $A$, so $\mathcal{R}_{A}$ is a subring of $R \otimes_{k} K$, where $K$ denotes the field of fractions of $A$. Then the image of $\mathcal{R}_{A, \lambda}$ in $R \otimes_{k} K$ is equal to $\pi^{h^{\prime}(\lambda)} \cdot V(\lambda) \otimes_{k} A \subset V(\lambda) \otimes_{k} K$ for some function $h^{\prime}: P \rightarrow \mathbb{Z}$.

Lemma 6.17. The function $h^{\prime}$ is admissible with respect to $(X, L)$ and defines a regular paving $S^{\prime}$ of $Q$ which is coarser than $S$.

Proof. Note that $h^{\prime}$ is linear on $P_{\omega}$ for each $\omega \in S$. Furthermore, if $V(\eta)$ occurs in $R_{\lambda} \cdot R_{\mu}$, then we must have $h^{\prime}(\lambda)+h^{\prime}(\mu) \geqslant h^{\prime}(\eta)$ since $\mathcal{R}$ is an algebra. It follows that the function $h^{\prime}$ is admissible and also that the associated paving $S^{\prime}$ is coarser than $S$.

6.18. Define $P_{S}^{\mathrm{gp}}$ and $P_{S^{\prime}}^{\mathrm{gp}}$ as in $\S 6.2$. Since $S^{\prime}$ is coarser than $S$, there is a surjection

$$
P_{S}^{\mathrm{gp}} \longrightarrow P_{S^{\prime}}^{\mathrm{gp}}
$$

which induces a surjection $\pi: K_{S} \rightarrow K_{S^{\prime}}$. 
6.19. Let

$$
\left(\mathscr{X}^{\prime}, M_{\mathscr{X}^{\prime}}, L_{\mathscr{X}^{\prime}}\right) \longrightarrow\left(\operatorname{Spec}\left(k\left[K_{S^{\prime}}\right]\right), M_{K_{S^{\prime}}}\right)
$$

be the polarized log scheme with $B$-action obtained from the construction of (6.12.3) using $S^{\prime}$ instead of $S$, and let $\left(Y^{\prime}, M_{Y^{\prime}}, L_{Y^{\prime}}\right) \rightarrow\left(\operatorname{Spec}(k), M_{k}^{\prime}\right)$ be the base change along the vertex morphism $k\left[K_{S^{\prime}}\right] \rightarrow k$.

Also let $\left(Y_{\beta}, M_{Y_{\beta}}, L_{Y_{\beta}}\right) /\left(\operatorname{Spec}(k), M_{\beta}\right)$ be the polarized log scheme obtained by pullback along $\beta$ of the family (6.12.3).

Proposition 6.20. There exist a commutative diagram

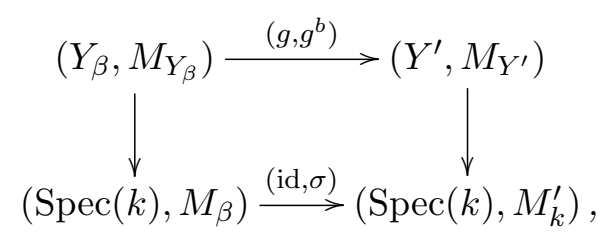

where the horizontal morphisms are isomorphisms, $\left(g, g^{b}\right)$ is $B$-equivariant, and $g$ is $G$-equivariant, and a $G$-equivariant isomorphism $g^{*} L_{Y^{\prime}} \simeq L_{Y_{\beta}}$.

Proof. Fix a map $\tilde{\beta}: K_{S} \rightarrow A$ to a discrete valuation ring $A$, as in $\S 6.16$. After possibly replacing $A$ by a finite extension, we can extend this to a map $K_{S}^{\text {sat }} \rightarrow A$, which we will again denote by $\tilde{\beta}$. Let $F \subset K_{S}$ denote the face generated by elements $\rho(\lambda)+\rho(\mu)-\rho(\lambda+\mu)$, where $\lambda, \mu \in P$ lie in a single $P_{\omega}$ for some $\omega \in S^{\prime}$, and let $K_{S, F}$ denote the localization of $K_{S}$ with respect to the face $F$.

Lemma 6.21. The surjection $K_{S} \rightarrow K_{S^{\prime}}$ induces an isomorphism $K_{S, F} / F^{\mathrm{gp}} \simeq K_{S^{\prime}}$.

Proof. Let $P_{S}$ and $P_{S^{\prime}}$ be as in $\S 6.2$. Then by the same argument as in [Ols08a, Proof of Lemma 3.1.21] the kernel of the surjection $P_{S}^{\mathrm{gp}} \rightarrow P_{S^{\prime}}^{\mathrm{gp}}$ is generated by elements $\rho(\lambda)+\rho(\mu)-$ $\rho(\lambda+\mu)$, where $\lambda$ and $\mu$ lie in a single $\omega \in S^{\prime}$. Since these elements already lie in $F$, this implies that the induced map

$$
K_{S, F} / F^{\mathrm{gp}} \longrightarrow K_{S^{\prime}}
$$

is injective. Since it is evidently surjective we obtain the lemma.

Proof of Proposition 6.20, continued. Note that we have $\tilde{\beta}(F) \subset A^{*}$. After possibly replacing $A$ by a finite extension once more, we can find a homomorphism $\gamma: K_{S}^{\mathrm{gp}} \rightarrow A^{*}$ whose restriction to $F^{\mathrm{gp}}$ is equal to the restriction of $\beta$. Let $\beta^{\prime}: K_{S} \rightarrow A$ be the map sending $h \in K_{S}$ to $\gamma(h)^{-1} \cdot \beta(h)$. Let $\mathcal{R}_{A}^{\prime}$ denote the base change along $\beta^{\prime}$ of $\mathcal{R}$.

Fix an extension $\tilde{\gamma}:\left(K_{S} \rtimes P\right)^{\mathrm{gp}} \rightarrow A^{*}$ of $\gamma$. Then the map

$$
\mathcal{R}_{A} \longrightarrow \mathcal{R}_{A^{\prime}}
$$

induced by multiplication by $\tilde{\gamma}(\lambda)$ on the $\lambda$-components is an isomorphism of $k\left[K_{S}\right]$-algebras. 


\section{OLSSON}

Furthermore, we have a commutative diagram

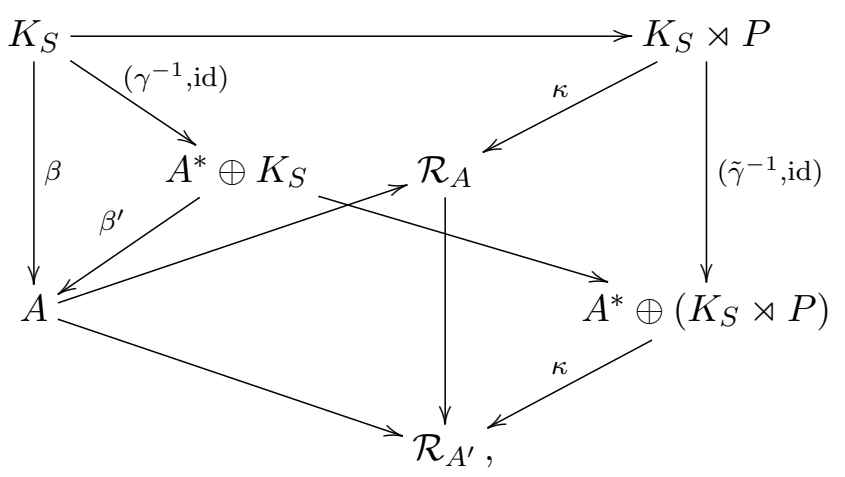

where the maps labeled $\kappa$ are the charts (6.12.1). From this it follows that it suffices to prove Proposition 6.20 after replacing $\beta$ by $\beta^{\prime}$. We may therefore assume $\beta(F)=1$, in which case the map $\tilde{\beta}$ factors through a map $\tilde{\beta}^{\prime}: K_{S^{\prime}} \rightarrow A$. Similarly, we may assume that the induced map $\tilde{\beta}^{\prime}: K_{S^{\prime}}^{\text {sat }} \rightarrow A$ sends $K_{S^{\prime}}^{\times}$to 1 , in which case the induced map $k\left[K_{S^{\prime}}\right] \rightarrow k$ is the vertex. In this case Proposition 6.20 follows immediately from the construction.

\section{Some calculations for the standard family}

In this section we collect various calculations of global sections that will be used in what follows.

7.1. Fix an irreducible polarized spherical variety $\left(X_{0}, L_{0}\right)$ over $k$ with moment polytope $Q$ and let $S$ be a paving of $Q$ admissible with respect to $\left(X_{0}, L_{0}\right)$. Let $\left(f, f^{b}\right):\left(X, M_{X}, L\right) \rightarrow$ $\left(\operatorname{Spec}(k), M_{k}\right)$ be the polarized log scheme obtained by base change from the standard construction along a morphism $K_{S} \rightarrow k$ factoring through a morphism $K_{S}^{\text {sat }} \rightarrow k$ sending all nonunits of $K_{S}^{\text {sat }}$ to 0 . Let $R$ (respectively, $\left.R_{0}\right)$ be the coordinate ring of the cone of $(X, L)$ (respectively, $\left.\left(X_{0}, L_{0}\right)\right)$. So if $\left(\widetilde{X}, M_{\widetilde{X}}\right) /\left(\operatorname{Spec}(k), M_{k}\right)$ denotes the base change of the log scheme $(6.12 .2)$, then $\widetilde{X}$ is affine with coordinate ring $R$.

To ease the notation, let $E$ denote the log scheme $\left(\operatorname{Spec}(k), M_{k}\right)$. Let $M_{X / E}$ be the relative characteristic of $\left(X, M_{X}\right) / E$. By definition this is the cokernel in the category of sheaves of integral monoids on $X$ of the morphism $f^{b}: f^{*} M_{E} \rightarrow M_{X}$. Similarly, define the relative characteristic $M_{\widetilde{X} / E}$ of $\left(\widetilde{X}, M_{\widetilde{X}}\right) /\left(\operatorname{Spec}(k), M_{k}\right)$.

Proposition 7.2. The natural map $P^{\mathrm{gp}} \rightarrow H^{0}\left(\widetilde{X}, M_{\widetilde{X} / E}^{\mathrm{gp}}\right)$ is an isomorphism.

The proof occupies 7.3-7.9.

7.3. For $\omega \in S$ we have a natural inclusion $P_{\omega} \hookrightarrow K_{S} \rtimes P$ which induces a morphism of $\log$ structures $M_{\omega} \hookrightarrow M_{X}$ on $X$, where $M_{\omega}$ is the $\log$ structure induced by the composition $P_{\omega} \rightarrow K_{S} \rtimes P \rightarrow R$. Let $R \rightarrow R_{\omega}$ be the quotient corresponding to the irreducible $\widetilde{G}$-subscheme of $\operatorname{Spec}(R)$ defined by $\omega$. So $R_{\omega, a}$ is nonzero if and only if $a$ lies in the integral points $P_{\omega}$ of the cone over $\omega$. Let $i_{\omega}: \widetilde{X}_{\omega} \hookrightarrow \widetilde{X}$ be the spectrum of $R_{\omega}$.

Note that

$$
\tilde{X}_{\omega} / / U:=\operatorname{Spec}\left(R_{\omega}^{U}\right)
$$




\section{MODULI OF SPHERICAL VARIETIES AND LOG GEOMETRY}

is an irreducible affine toric variety, and $\left.M_{\omega}\right|_{\widetilde{X}_{\omega}}$ is the pullback of the standard log structure on this toric variety.

We have an exact sequence of sheaves of groups on $\widetilde{X}_{\omega}$

$$
\left.\left.0 \longrightarrow \bar{M}_{\omega}^{\mathrm{gp}}\right|_{\widetilde{X}_{\omega}} \longrightarrow M_{\widetilde{X} / E}^{\mathrm{gp}}\right|_{\widetilde{X}_{\omega}} \longrightarrow P^{\mathrm{gp}} / P_{\omega}^{\mathrm{gp}} \longrightarrow 0
$$

where $P^{\mathrm{gp}} / P_{\omega}^{\mathrm{gp}}$ denotes the constant sheaf. To prove Proposition 7.2 it suffices to show that the map

$$
P_{\omega}^{\mathrm{gp}} \longrightarrow H^{0}\left(\widetilde{X}_{\omega},\left.\bar{M}_{\omega}^{\mathrm{gp}}\right|_{\widetilde{X}_{\omega}}\right)
$$

is an isomorphism. For then using (7.3.1) we get that the map

$$
P^{\mathrm{gp}} \longrightarrow H^{0}\left(\widetilde{X}_{\omega},\left.M_{\widetilde{X} / E}^{\mathrm{gp}}\right|_{\widetilde{X}_{\omega}}\right)
$$

is an isomorphism. Now choose an ordering $\left\{\omega_{1}, \ldots, \omega_{r}\right\}$ of the top-dimensional simplices of $S$ and for two indices $i, j$ let $\omega_{i j}$ denote the intersection $\omega_{i} \cap \omega_{j}$. Then we have an exact sequence of sheaves on $\tilde{X}$

$$
\left.\left.0 \longrightarrow M_{\tilde{X} / E}^{\mathrm{gp}} \longrightarrow \prod_{j=1}^{r} i_{\omega_{j} *} M_{\tilde{X} / E}^{\mathrm{gp}}\right|_{\widetilde{X}_{\omega_{j}}} \longrightarrow \prod_{j<k} i_{\omega_{j k *}} M_{\widetilde{X} / E}^{\mathrm{gp}}\right|_{\widetilde{X}_{\omega_{j k}}}
$$

Taking global sections, we then get Proposition 7.2 in general.

It therefore suffices to prove Proposition 7.2 in the case when $X$ is irreducible, which we assume henceforth. Note that in this case $M_{X / E}=\bar{M}_{X}$.

Lemma 7.4. Let $X$ be a finite type $k$-scheme and let $\Lambda$ be a torsion Gorenstein local ring of dimension 0 . Let $r \geqslant 0$ be an integer and let $f: X \times \mathbb{A}^{r} \rightarrow X$ be the first projection. Then for any $F$ in the derived category $D_{c}^{b}(X, \Lambda)$ of bounded complexes of $\Lambda$-modules on the étale site of $X$ with constructible cohomology sheaves, the adjunction map

$$
F \longrightarrow R f_{*} f^{*} F
$$

is an isomorphism.

Proof. Using Verdier duality, it suffices to show the dual statement: for any $F \in D_{c}^{b}(X, \Lambda)$ the adjunction map

$$
R f_{!} f^{!} F \longrightarrow F
$$

is an isomorphism. Note that $f^{!} F \simeq f^{*} F(r)[2 r]$ since $f$ is smooth of relative dimension $r$. By the base change theorem for $R f_{\text {! }}$ we can verify this after base changing to a point. Applying duality again over a point this then reduces the proof to the case when $X=\operatorname{Spec}(k)$, where the result is immediate.

Remark 7.5. Lemma 7.4 remains true (with the same proof) over fields of positive characteristic invertible in $\Lambda$.

For a $k$-scheme $T$, let $\widetilde{X}_{T}$ denote the base change of $\widetilde{X}$ to $T$ and let $M_{\widetilde{X}_{T}}$ denote the pullback of the $\log$ structure $M_{\widetilde{X}}$ to $\widetilde{X}_{T}$.

Lemma 7.6. For any $k$-scheme $T$ the action of $U(T)$ on $H^{0}\left(\widetilde{X}_{T}, \bar{M}_{\widetilde{X}_{T}}^{\mathrm{gp}}\right)$ is trivial. 


\section{OLSSON}

Proof. For an integer $N$ let $F_{N}$ denote the sheaf $\bar{M}_{\widetilde{X}_{T}}^{\mathrm{gp}} / N \bar{M}_{\widetilde{X}_{T}}^{\mathrm{gp}}$. It suffices to show that the $U(T)$-action on each $H^{0}\left(\widetilde{X}_{T}, F_{N}\right)$ is trivial. Consider the diagram

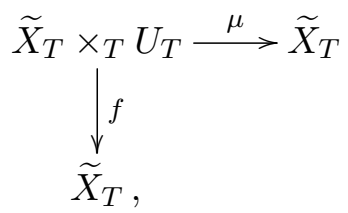

where the map $f$ is the projection and the map $\mu$ is the action. This is a diagram of schemes over $(X / / U)_{T}$, and since $F_{N}$ is pulled back from $(X / / U)_{T}$, we get an isomorphism $\epsilon: \mu^{*} F_{N} \rightarrow f^{*} F_{N}$.

For a point $u \in U(T)$ the action on $H^{0}\left(\widetilde{X}_{T}, F_{N}\right)$ is then the composite map

$$
H^{0}\left(\widetilde{X}_{T}, F_{N}\right) \stackrel{\mu^{*}}{\longrightarrow} H^{0}\left(\widetilde{X}_{T} \times_{T} U_{T}, \mu^{*} F_{N}\right) \stackrel{\epsilon}{\longrightarrow} H^{0}\left(\widetilde{X}_{T} \times_{T} U_{T}, f^{*} F_{N}\right) \stackrel{(\mathrm{id} \times u)^{*}}{\longrightarrow} H^{0}\left(\widetilde{X}_{T}, F_{N}\right) .
$$

Now observe that the map

$$
H^{0}\left(\widetilde{X}_{T} \times_{T} U_{T}, f^{*} F_{N}\right) \stackrel{(\mathrm{id} \times u)^{*}}{\longrightarrow} H^{0}\left(\widetilde{X}_{T}, F_{N}\right)
$$

is inverse to the pullback map $f^{*}: H^{0}\left(\widetilde{X}_{T}, F_{N}\right) \rightarrow H^{0}\left(\widetilde{X}_{T} \times_{T} U_{T}, f^{*} F_{N}\right)$, which is an isomorphism by Lemma 7.4. In particular, the composite map (7.6.1) is independent of the choice of $u$. Taking $u$ the identity element of $U$, we obtain the lemma.

Remark 7.7. It follows that we get an action of $U$ on $H^{0}\left(\widetilde{X}, M_{\widetilde{X}}^{\mathrm{gp}}\right)$. Indeed, for any $m \in$ $H^{0}\left(\widetilde{X}, M_{\widetilde{X}}^{\mathrm{gp}}\right)$ and a point $u \in U(T)$ over some scheme $T$, there exists a unique unit $v_{m}(u) \in$ $\Gamma\left(\widetilde{X}_{T}, \mathscr{O}_{\widetilde{X}_{T}^{*}}^{*}\right)=\Gamma\left(T, \mathscr{O}_{T}^{*}\right)$ for which in $H^{0}\left(\widetilde{X}_{T}, M_{\widetilde{X}_{T}}^{\mathrm{gp}}\right)$ we have $u^{*} m=v_{m}(u)+m$. This in fact defines a morphism of group schemes $v_{m}: U \rightarrow \mathbb{G}_{m}$ which must be trivial since $U$ is unipotent. It follows that the action of $U$ on $H^{0}\left(\widetilde{X}, M_{\widetilde{X}}^{\mathrm{gp}}\right)$ is also trivial.

Let $j: V \hookrightarrow \widetilde{X} / / U$ be the locus of triviality of the $\log$ structure on $\widetilde{X} / / U$ (so $V$ is isomorphic to a torus), and let $\tilde{j}: \widetilde{X}_{V} \hookrightarrow \widetilde{X}$ be the preimage.

LEMma 7.8. The natural maps

$$
k^{*} \oplus P^{\mathrm{gp}} \longrightarrow H^{0}\left(\widetilde{X}_{V}, \mathscr{O}_{\widetilde{X}_{V}}^{*}\right)^{U(k)} \quad \text { and } \quad P^{\mathrm{gp}} \longrightarrow H^{0}\left(\widetilde{X},\left(\tilde{j}_{*} \mathscr{O}_{\widetilde{X}_{V}}^{*} / k^{*}\right)\right)^{U(k)}
$$

are isomorphisms.

Proof. Note first of all that since $k^{*}$ is a constant sheaf and $\widetilde{X}$ is irreducible, the map

$$
H^{0}\left(\widetilde{X}_{V}, \mathscr{O}_{\widetilde{X}_{V}}^{*}\right) \longrightarrow H^{0}\left(\widetilde{X},\left(\tilde{j}_{*} \mathscr{O}_{\widetilde{X}_{V}^{*}}^{*} / k^{*}\right)\right)
$$

is surjective. We claim that it remains surjective after taking $U$-invariants. To see this, let $\bar{m} \in H^{0}\left(\widetilde{X},\left(\tilde{j}_{*} \mathscr{O}_{\widetilde{X}_{V}^{*}}^{*} / k^{*}\right)\right)$ be a $U(k)$-invariant section, and let $T_{\bar{m}}$ be the set of liftings of $\bar{m}$ to $H^{0}\left(\widetilde{X}_{V}, \mathscr{O}_{\widetilde{X}_{V}}^{*}\right)$. The set $T_{\bar{m}}$ has a simply transitive action of $k^{*}$ and a commuting $U(k)$-action. To prove the lemma it suffices to show that $T_{\bar{m}}$, with its $k^{*}$ - and $U(k)$-actions, is the set of $k$ points of a $\mathbb{G}_{m}$-torsor with $U$-action. For such torsors are classified by homomorphisms $U \rightarrow \mathbb{G}_{m}$, and all such homomorphisms are trivial since $U$ is unipotent.

Consider the inclusion of sets with $k^{*}$ - and $U(k)$-action

$$
H^{0}\left(\widetilde{X}_{V}, \mathscr{O}_{\widetilde{X}_{V}}^{*}\right) \longleftrightarrow H^{0}\left(\widetilde{X}_{V}, \mathscr{O}_{\widetilde{X}_{V}}\right)
$$




\section{MODULI OF SPHERICAL VARIETIES AND LOG GEOMETRY}

Let $R_{V}$ denote the coordinate ring of $\widetilde{X}_{V}$. We can then consider the sheaf $\mathscr{A}$ on the big étale site of $\operatorname{Spec}(k)$ associating to any $k$-algebra $B$ the $\operatorname{ring} R_{V} \otimes_{k} B$. This is a sheaf with $U$-action and we also have an action of $\mathbb{G}_{m}$ on this sheaf. Let $\mathscr{Q}$ denote the quotient, in the category of sheaves on the big étale site of $\operatorname{Spec}(k)$, of $\mathscr{A}$ by the $\mathbb{G}_{m}$-action. The section $\bar{m}$ then defines a global section $q_{\bar{m}} \in H^{0}(\operatorname{Spec}(k), \mathscr{Q})$ and $T_{\bar{m}}$ is the set of $k$-points of the $\mathbb{G}_{m}$-torsor of liftings of $q_{\bar{m}}$ to $\mathscr{A}$.

It follows that it suffices to show that the map $k^{*} \oplus P^{g p} \rightarrow \Gamma\left(\widetilde{X}_{V}, \mathscr{O}_{\widetilde{X}_{V}}^{*}\right)^{U(k)}$ is an isomorphism. This follows from noting that $\Gamma\left(\widetilde{X}_{V}, \mathscr{O}_{\widetilde{X}_{V}}^{*}\right)^{U(k)}=\Gamma\left(V, \mathscr{O}_{V}^{*}\right)$ and that $V$ is isomorphic to the torus associated to $P^{\text {gp }}$.

7.9. To complete the proof of Proposition 7.2 observe that the map $M_{X} \rightarrow \mathscr{O}_{\widetilde{X}}$ induces an inclusion of sheaves

and therefore also an inclusion

$$
\bar{M}_{X}^{\mathrm{gp}} \longleftrightarrow\left(\tilde{j}_{*} \mathscr{O}_{\widetilde{X}_{V}}^{*}\right) / k^{*}
$$

$$
\theta: H^{0}\left(\widetilde{X}, \bar{M}_{\widetilde{X}}^{\mathrm{gp}}\right) \hookrightarrow H^{0}\left(\widetilde{X},\left(\tilde{j}_{*} \mathscr{O}_{\widetilde{X}_{V}}^{*}\right) / k^{*}\right)^{U(k)}=P^{\mathrm{gp}} .
$$

Since the composite of $\theta$ with the map $\rho: P^{\mathrm{gp}} \rightarrow H^{0}\left(\widetilde{X}, \bar{M}_{\widetilde{X}}^{\mathrm{gp}}\right)$ is the identity on $P^{\mathrm{gp}}$, this implies that $\rho$ is an isomorphism.

This completes the proof of Proposition 7.2.

Let $\widetilde{X}^{\circ}$ denote the complement of the vertex in $\widetilde{X}$.

Lemma 7.10. (i) The natural map $P^{\mathrm{gp}} \rightarrow H^{0}\left(\widetilde{X}^{\circ}, M_{\widetilde{X} / E}^{\mathrm{gp}}\right)$ is an isomorphism.

(ii) The map $P^{\mathrm{gp}} \rightarrow H^{0}\left(X, M_{X / E}^{\mathrm{gp}}\right)$ is an isomorphism.

Proof. Statement (i) follows from the same reasoning as that proving Proposition 7.2, replacing $\widetilde{X}$ by $\widetilde{X}^{\circ}$. Statement (ii) follows from statement (i), after noting that there is an inclusion $H^{0}\left(X, M_{X / E}^{\mathrm{gp}}\right) \hookrightarrow H^{0}\left(\widetilde{X}^{\circ}, M_{\tilde{X} / E}^{\mathrm{gp}}\right)$ compatible with the maps from $P^{\mathrm{gp}}$.

Remark 7.11. A variant of the argument proving Proposition 7.2 also shows that the restriction map

is an isomorphism.

$$
H^{0}\left(\widetilde{X}, M_{\widetilde{X} / E}\right) \longrightarrow H^{0}\left(\widetilde{X}^{\circ}, M_{\widetilde{X}^{\circ} / E}\right)
$$

Remark 7.12. By the same argument as in [Ols08a, Lemma 3.3.7] the restriction map

$$
\Gamma\left(\widetilde{X}, \mathscr{O}_{\widetilde{X}}\right) \longrightarrow \Gamma\left(\widetilde{X}^{\circ}, \mathscr{O}_{\widetilde{X}^{\circ}}\right)
$$

is an isomorphism. The isomorphism $P^{\mathrm{gp}} \rightarrow H^{0}\left(X, M_{X / E}^{\mathrm{gp}}\right)$ is characterized by the property that for any $p \in P$ the image of $p$ in $H^{0}\left(X, M_{X / E}^{\text {gp }}\right)$ admits a lifting to $H^{0}\left(X, M_{X}\right)$ whose image in $\Gamma\left(\widetilde{X}^{\circ}, \mathscr{O}_{\widetilde{X}^{\circ}}\right) \simeq \Gamma\left(\widetilde{X}, \mathscr{O}_{\widetilde{X}}\right)$ maps to a highest weight vector for the representation corresponding to $p$.

Lemma 7.13. Let $\alpha \in P^{\text {gp }}$ be an element not in $P$, and let $K_{S}^{\prime}$ denote the image of $K_{S}$ in $K_{S}^{\text {sat }} / K_{S}^{\text {sat* }}$. Then there exist morphisms of monoids $h: K_{S}^{\prime} \rtimes P \rightarrow \mathbb{N}$ and $g: P \rightarrow \mathbb{N}$ such that the following hold:

(i) $h\left(K_{S}^{\prime}-\{0\}\right) \subset \mathbb{N}_{>0}$. 


\section{OLSSON}

(ii) There exists a nonzero element $p \in P$ such that $h(0, p)=0$ and $g(p)=0$.

(iii) We have $g^{\mathrm{gp}}(\alpha)<0$, where $g^{\mathrm{gp}}: P^{\mathrm{gp}} \rightarrow \mathbb{Z}$ is the map induced by $g$.

Proof. Recall [Kat94, Proposition 5.8(1)] that we have

$$
P=\bigcap_{F} P_{F}
$$

where the intersection runs over the faces of codimension 1 of $P$. We can therefore find a face $F$ of the polytope $Q$ such that $\alpha$ does not lie in $P_{F}$. By duality for monoids [Ogu14, Theorem 2.2.3] we can then find a homomorphism $g: P \rightarrow \mathbb{N}$ sending $F$ to 0 and such that $g^{\mathrm{gp}}(\alpha)<0$. Note that the subset $\{0\} \times F \subset K_{S}^{\prime} \rtimes P$ is also a face. We can therefore find a homomorphism

$$
h: K_{S}^{\prime} \rtimes P \longrightarrow \mathbb{N}
$$

sending $\{0\} \times F$ to 0 and $K_{S}^{\prime}-\{0\}$ to $\mathbb{N}_{>0}$.

Lemma 7.14. The diagram

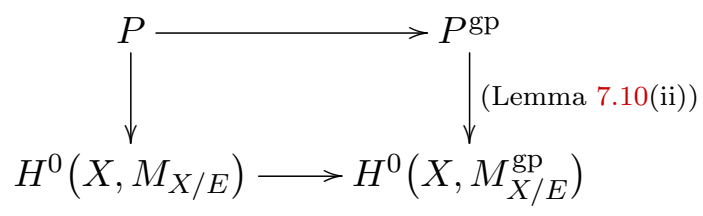

is cartesian, so in particular the natural map $P \rightarrow H^{0}\left(X, M_{X / E}\right)$ is an isomorphism.

Proof. Let $\alpha \in P^{\mathrm{gp}}$ be an element not in $P$ whose image in $H^{0}\left(X, M_{X / E}^{\mathrm{gp}}\right)$ lies in $H^{0}\left(X, M_{X / E}\right)$. We show that this gives a contradiction. Choose $h: K_{S} \rtimes P \rightarrow \mathbb{N}$ and $g: P \rightarrow \mathbb{N}$ as in Lemma 7.13. Let $N$ denote the $\log$ structure $k^{*} \oplus \mathbb{N} \oplus \mathbb{N}$ on $\operatorname{Spec}(k)$ sending all nonzero elements of $\mathbb{N}^{2}$ to 0 in $k$. We then have a commutative diagram of monoids

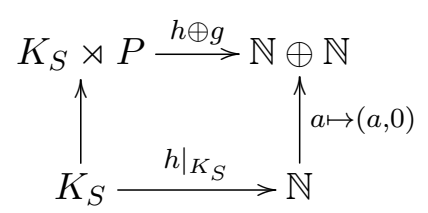

such that the induced morphism on relative characteristics $P \rightarrow \mathbb{N}$ is the map $g$. This diagram induces a morphism of $\log$ schemes

$$
(\operatorname{Spec}(k), N) \longrightarrow\left(\operatorname{Spec}\left(R^{U}\right), M_{R^{U}}\right)
$$

over $\left(\operatorname{Spec}(k), M_{k}\right)$. As discussed in $\left[\mathrm{AB} 05\right.$, proof of Lemma 1.2] the map $\operatorname{Spec}(R) \rightarrow \operatorname{Spec}\left(R^{U}\right)$ is surjective. We can therefore lift this point to a morphism of log schemes

$$
(\operatorname{Spec}(k), N) \longrightarrow\left(\tilde{X}, M_{\tilde{X}}\right)
$$

The image of this point is not the vertex of $\widetilde{X}$ since $g$ sends a nonzero face to 0 in $\mathbb{N}$. We therefore get a morphism of log schemes

$$
\left(t, t^{b}\right):(\operatorname{Spec}(k), N) \longrightarrow\left(X, M_{X}\right)
$$

such that the induced map

$$
P^{\mathrm{gp}} \simeq H^{0}\left(X, M_{X / E}^{\mathrm{gp}}\right) \longrightarrow\left(N / M_{k}\right)^{\mathrm{gp}} \simeq \mathbb{Z}
$$

is the map $g$ and sends $H^{0}\left(X, M_{X / E}\right)$ to $\mathbb{N}$. This gives a contradiction since $g(\alpha)<0$. 


\section{MODULI OF SPHERICAL VARIETIES AND LOG GEOMETRY}

\section{Enriched log structures}

8.1. Let $T$ be a $k$-scheme and consider a collection of data

$$
\left(M_{T},\left(f, f^{b}\right):\left(X, M_{X}\right) \longrightarrow\left(T, M_{T}\right),\left(\rho, \rho^{b}\right), L\right)
$$

as follows:

(i) $M_{T}$ is a fine $\log$ structure on $T$.

(ii) $\left(f, f^{b}\right):\left(X, M_{X}\right) \rightarrow\left(T, M_{T}\right)$ is a morphism of fine log schemes whose underlying morphism of schemes $f$ is proper, locally of finite presentation, and flat.

(iii) $\rho$ is an action of $G$ on $X / T$, and $\rho^{b}$ is an action of $B$ on $\left(X, M_{X}\right)$ over $\left(T, M_{T}\right)$ such that the underlying action of $B$ on $X$ is equal to the action obtained by restriction from $G$.

(iv) $L$ is a $G$-linearized ample invertible sheaf on $X$ such that $(X, L)$ is a stable spherical variety over $T$.

(v) For every geometric point $\bar{t} \rightarrow T$ the pullback of the collection (8.1.1) to $\bar{t}$ is isomorphic to that obtained by the standard construction from some starting variety $\left(X_{0}, L_{0}\right)$ and some paving regular with respect to $\left(X_{0}, L_{0}\right)$.

Let $\mathcal{R}$ denote the quasi-coherent sheaf of $\mathscr{O}_{T}$-algebras given by the relative cone of $(X, L)$, and for $a \in P$ let $\mathcal{R}_{a}$ denote the corresponding $\mathscr{O}_{T}$-submodule of $\mathcal{R}$. Let $\tilde{f}: \tilde{X} \rightarrow T$ denote the relative spectrum of $\mathcal{R}$, and let $\tilde{f}^{\circ}: \widetilde{X}^{\circ} \rightarrow T$ denote the complement of the vertex, so that $X$ is the quotient of $\widetilde{X}^{\circ}$ by the natural $\mathbb{G}_{m}$-action. Let $M_{\widetilde{X}^{\circ}}$ denote the pullback of $M_{X}$.

By the proper base change theorem and Lemma 7.14, the stalk of $f_{*} M_{X / T}$ is isomorphic to $P$ at every geometric point. Assume in addition to the above that the following conditions hold:

(vi) There exists an isomorphism $P \rightarrow f_{*} M_{X / T}$ inducing the isomorphism of Lemma 7.14 in every geometric fiber.

(vii) The natural map $\tilde{f}_{*}^{\circ} M_{\widetilde{X}^{\circ}} \rightarrow f_{*} M_{X / T}$ is surjective, and the same remains true after arbitrary base change $T^{\prime} \rightarrow T$.

Remark 8.2. Note that it follows from condition (vi) that the pullback map $f_{*} M_{X / T} \rightarrow \tilde{f}_{*}^{\circ} M_{\widetilde{X}^{\circ} / T}$ is an isomorphism. The map $\tilde{f}_{*}^{\circ} M_{\widetilde{X}^{\circ}} \rightarrow f_{*} M_{X / T}$ in condition (vii) is the one induced from this and the map $\tilde{f}_{*}^{\circ} M_{\widetilde{X}^{\circ}} \rightarrow \tilde{f}_{*}^{\circ} M_{\widetilde{X}^{\circ} / T}$ arising from the quotient map $M_{\widetilde{X}^{\circ}} \rightarrow M_{\widetilde{X}^{\circ} / T}$.

8.3. The discussion of $\S 2.5$ then applies and we get for every element $p \in P$ an $\mathscr{O}_{T}^{*}$-torsor $L_{p}$ and for any two elements $p, q \in P$ a map

$$
\theta_{p, q}: L_{p} \wedge L_{q} \longrightarrow M_{T} \wedge L_{p+q}
$$

LEMMA 8.4. The restriction map

$$
\mathcal{R} \longrightarrow \tilde{f}_{*}^{\circ} \mathscr{O}_{\widetilde{X}^{\circ}}
$$

is an isomorphism.

Proof. Since $X=\left[\widetilde{X}^{\circ} / \mathbb{G}_{m}\right]$, we have

$$
\tilde{f}_{*}^{\circ} \mathscr{O}_{\widetilde{X}^{\circ}}=\oplus_{n \in \mathbb{Z}} f_{*} L^{\otimes n} .
$$

By [AB06, paragraph following Definition 3.2.1] we have

$$
\mathcal{R} \simeq \oplus_{n \geqslant 0} f_{*} L^{\otimes n},
$$




\section{OLSSON}

so to prove the lemma it suffices to show $f_{*} L^{\otimes n}=0$ for $n<0$, which follows from Remark 7.12, which shows it holds in every fiber.

8.5. For $a \in P$ let

$$
\alpha_{a}: L_{a} \longrightarrow \mathcal{R}_{a}
$$

denote the map induced by the map

$$
\tilde{f}_{*}^{\circ} M_{\widetilde{X}^{\circ}} \stackrel{\alpha_{M}}{\longrightarrow} \tilde{f}_{*}^{\circ} \mathscr{O}_{\widetilde{X}^{\circ}} \simeq \mathcal{R}
$$

Definition 8.6. A logarithmic enrichment $\underline{\eta}=\left\{\eta_{a, b}^{c}\right\}$ on the family (8.1.1) is a collection of maps

$$
\eta_{a, b}^{c}: L_{a} \wedge L_{b} \longrightarrow M_{T} \wedge L_{c}
$$

one for each triple $a, b, c \in P$ with $(a, b) \succ c$, such that the following conditions hold:

(i) For all $a, b \in P$ we have $\eta_{a, b}^{a+b}=\theta_{a, b}$.

(ii) For all $(a, b) \succ c$ we have $\eta_{a, b}^{c}=\eta_{b, a}^{c}$.

(iii) For all $a, b, c, d, e, f \in P$ (some of them possibly equal) with

$$
e \prec(a, b), d \prec(e, c), f \prec(b, c) \text {, and } d \prec(a, f)
$$

the diagram

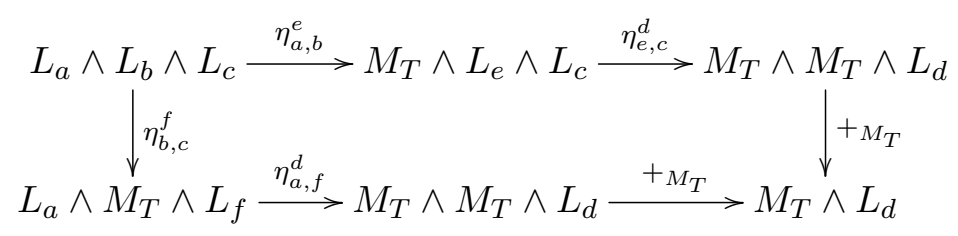

commutes.

(iv) For all $a, b, c, d, e, f \in P$ with

$$
e \prec(a, b), d \prec(c, e), f \prec(a, b), d \prec(c, f)
$$

the diagram

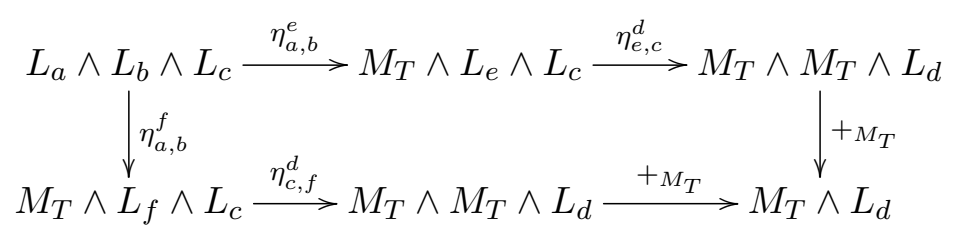

commutes.

(v) For every $c \prec(a, b)$ the diagram

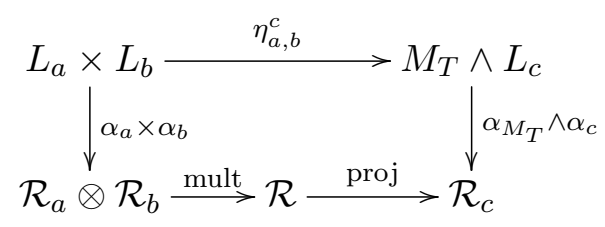

commutes, where proj: $\mathcal{R} \rightarrow \mathcal{R}_{c}$ is the projection. 


\section{MODULI OF SPHERICAL VARIETIES AND LOG GEOMETRY}

8.7. With notation as in $\S 6.1$, the standard family (6.12.3) over $k\left[K_{S}\right]$ has a canonical logarithmic enrichment $\eta$. Indeed, in this case the torsor $L_{p}$ is the $\mathbb{G}_{m}$-torsor of highest weight vectors in $\mathcal{R}_{p}$ (notation as in $\S 6.12$ ). In particular, we have a trivialization $v_{p}$ for $\mathcal{R}_{p}$. For $c \prec(a, b)$ the map

$$
\eta_{a, b}^{c}: L_{a} \wedge L_{b} \longrightarrow M_{K_{S}} \wedge L_{c}
$$

is given by sending $v_{a} \wedge v_{b}$ to the image of $a+b-c \in K_{S}$ in $M_{K_{S}}$ times $v_{c}$. The fact that this defines a logarithmic enrichment follows immediately from the construction.

8.8. Assume now that $T=\operatorname{Spec}(A)$ is the spectrum of an $\operatorname{artinian}$ local $\operatorname{ring} A$ with residue field $\kappa$, and that the closed fiber

$$
\left(M_{\kappa},\left(f_{\kappa}, f_{\kappa}^{b}\right):\left(X_{\kappa}, M_{X_{\kappa}}\right) \longrightarrow\left(\operatorname{Spec}(\kappa), M_{\kappa}\right),\left(\rho_{\kappa}, \rho_{\kappa}^{b}\right), L_{\kappa}\right)
$$

of (8.1.1) is isomorphic to the base change of the standard family associated to a paving $S$ of $Q$ and a morphism $\beta_{\kappa}: K_{S} \rightarrow \kappa$ induced by a morphism $K_{S}^{\text {sat }} \rightarrow \kappa$ sending all nonunits to 0 . Assume further given a logarithmic enrichment $\eta=\left\{\eta_{a, b}^{c}\right\}$ on (8.1.1) reducing to the standard enrichment on the closed fiber (8.8.1) arising from its description as a standard family.

Proposition 8.9. There exists a homomorphism $\beta: K_{S} \rightarrow A$ such that the family (8.1.1) together with its enrichment $\eta$ is isomorphic, as a deformation of the closed fiber, to the base change along $\beta$ of the standard family.

We will in fact prove a slightly stronger statement (Proposition 8.11) that we will need later.

8.10. Let $A \rightarrow A_{0}$ be a surjective map of artinian local rings with kernel $J \subset A$ annihilated by the maximal ideal of $A$, so $J$ can be viewed as a $\kappa$-vector space. Assume given a morphism $\beta_{A_{0}}: K_{S} \rightarrow A_{0}$ lifting $\beta_{\kappa}$, so that the family over $A_{0}$ is isomorphic to the base change of the standard family along $\beta_{A_{0}}$, and fix such an isomorphism.

Proposition 8.11. There exists a morphism $\beta_{A}: K_{S} \rightarrow A$ lifting $\beta_{A_{0}}$ such that the family over $A$ is isomorphic to the base change of the standard family along $\beta_{A}$.

Remark 8.12. Note that if we proceed by induction on the length of $A$, Proposition 8.11 implies Proposition 8.9.

The proof of Proposition 8.11 occupies the remainder of this section.

Lemma 8.13. (i) The map $H^{0}\left(\widetilde{X}_{A}^{\circ}, M_{\widetilde{X}_{A}^{\circ}}\right) \longrightarrow H^{0}\left(\widetilde{X}_{A_{0}}^{\circ}, M_{\widetilde{X}_{A_{0}}^{\circ}}\right)$ is surjective.

(ii) The action of $U$ on $H^{0}\left(\widetilde{X}_{A}^{\circ}, M_{\widetilde{X}_{A}^{\circ}}\right)$ is trivial.

Proof. It suffices to prove the analogous results with $M_{\widetilde{X}_{A}}$ (respectively, $M_{\widetilde{X}_{A_{0}}}$ ) replaced by $M_{\widetilde{X}_{A}}^{\text {gp }}$ (respectively, $M_{\widetilde{X}_{A_{0}}}^{\mathrm{gp}}$ ). For this note that by assumption 8.1 (vii) we have a commutative diagram with exact rows

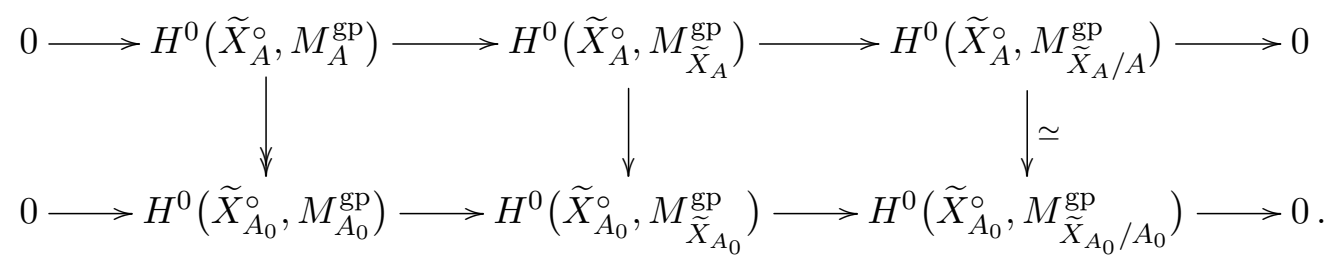




\section{OLSSON}

From this and the snake lemma, statement (i) follows.

Statement (ii) follows from a similar argument as in Remark 7.7.

Let $\gamma_{A_{0}}: K_{S} \rtimes P \rightarrow H^{0}\left(\widetilde{X}_{A_{0}}^{\circ}, M_{\widetilde{X}_{A_{0}}^{\circ}}\right)$ be the chart obtained from the identification of the family over $A_{0}$ with the standard family.

LEMma 8.14. The chart $\gamma_{A_{0}}$ lifts to a chart $\gamma_{A}: K_{S} \rtimes P \rightarrow H^{0}\left(\widetilde{X}_{A}^{\circ}, M_{\widetilde{X}_{A}^{\circ}}\right)$.

Proof. It suffices to show that the map $\gamma_{A_{0}}$ lifts to a map $K_{S} \rtimes P \rightarrow H^{0}\left(\widetilde{X}_{A}^{\circ}, M_{\widetilde{X}_{A}^{\circ}}^{\mathrm{gp}}\right)$.

From the exact sequence of sheaves

$$
0 \longrightarrow 1+J \otimes \mathscr{O}_{\widetilde{X}_{\kappa}^{\circ}} \longrightarrow M_{\widetilde{X}_{A}^{\circ}}^{\mathrm{gp}} \longrightarrow M_{\widetilde{X}_{A_{0}}^{\circ}}^{\mathrm{gp}} \longrightarrow 0
$$

we get a diagram

$$
0 \longrightarrow H^{0}\left(\widetilde{X}_{\kappa}^{\circ}, J \otimes \mathscr{O}_{\widetilde{X}_{\kappa}^{\circ}}\right) \longrightarrow H^{0}\left(\widetilde{X}_{A}^{\circ}, M_{\widetilde{X}_{A}^{\circ}}^{\mathrm{gp}}\right) \longrightarrow H_{-K_{S} \rtimes P .}\left(\widetilde{X}_{A_{0}}^{\circ}, M_{\widetilde{X}_{A_{0}}^{\circ}}^{\mathrm{gp}}\right) \longrightarrow H^{1}\left(\widetilde{X}_{\kappa}^{\circ}, J \otimes \mathscr{O}_{\widetilde{X}_{\kappa}^{\circ}}\right)
$$

Since $H^{0}\left(\widetilde{X}_{\kappa}^{\circ}, J \otimes \mathscr{O}_{\widetilde{X}_{\kappa}^{\circ}}\right)$ is a $\mathbb{Q}$-vector space, to prove the existence of the dotted arrow it suffices to show that the map $\epsilon$ is 0 . Let $z \in K_{S} \rtimes P$ be an element. The class $\epsilon(z)$ is the class of the torsor of liftings of $\gamma_{A_{0}}(z)$ to $M_{\widetilde{X}_{A}^{o}}$. Considering the commutative diagram

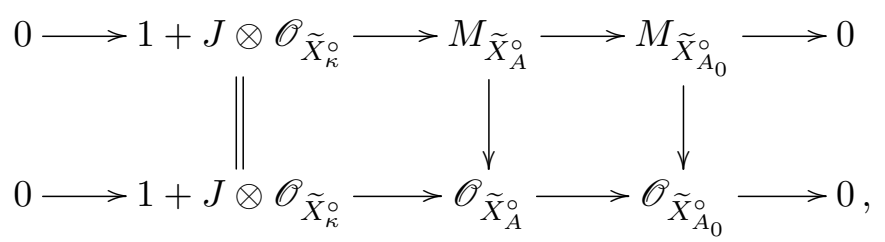

we get that the torsor corresponding to $\epsilon(z)$ is equal to the torsor of liftings of $\alpha\left(\gamma_{A_{0}}(z)\right) \in$ $H^{0}\left(\widetilde{X}_{A_{0}}^{\circ}, \mathscr{O}_{\widetilde{X}_{A_{0}}^{\circ}}\right)$ to $\mathscr{O}_{\widetilde{X}_{A}^{\circ}}$. Since the section $\alpha\left(\gamma_{A_{0}}(z)\right)$ extends to $\widetilde{X}_{A_{0}}$ by Lemma 8.4 , it follows that the class $\epsilon(z)$ is in the image of the restriction map

$$
H^{1}\left(\widetilde{X}_{\kappa}, J \otimes \mathscr{O}_{\widetilde{X}_{\kappa}}\right) \longrightarrow H^{1}\left(\widetilde{X}_{\kappa}^{\circ}, J \otimes \mathscr{O}_{\widetilde{X}_{\kappa}^{\circ}}\right)
$$

whence 0 since $\widetilde{X}_{\kappa}$ is affine.

8.15. Fix a lifting $\gamma_{A}$ of $\gamma_{A_{0}}$. By Lemma 8.13(ii) this lifting induces a morphism $K_{S} \rtimes P \rightarrow R_{A}^{U}$ and therefore a $T$-equivariant $\log$ structure on $\operatorname{Spec}\left(R_{A}^{U}\right)$ lifting the $\log$ structure on $\operatorname{Spec}\left(R_{A_{0}}^{U}\right)$. Let $\beta_{A}: K_{S} \rightarrow M_{A}$ be the restriction of $\gamma_{A}$, so $\beta_{A}$ is a lifting of the chart $\beta_{A_{0}}$.

For $a \in P$ let $L_{a, A}$ be the torsor defined as in $\S 8.3$. The map $\gamma_{A}$ defines a trivialization of these torsors $L_{a, A}$ lifting the standard trivializations over $A_{0}$. In particular, the maps $\eta_{a, b}^{c}$ for $c \prec(a, b)$ become identified with elements $\tilde{\eta}_{a, b}^{c} \in \Gamma\left(\operatorname{Spec}(A), M_{A}\right)$, lifting the corresponding elements over $A_{0}$. It follows that $\tilde{\eta}_{a, b}^{c}$ can be written uniquely as $u_{a, b}^{c}$ plus the image under $\beta$ of the element $\rho(a)+\rho(b)-\rho(c) \in K_{S}$, where $u_{a, b}^{c}$ is an element of

$$
\operatorname{Ker}\left(A^{*} \longrightarrow A_{0}^{*}\right)=1+J \text {. }
$$

Let $E$ be as in $\S 5.3$. 


\section{MODULI OF SPHERICAL VARIETIES AND LOG GEOMETRY}

Lemma 8.16. The elements $u_{a, b}^{c}$ define a homomorphism $u: E \rightarrow J$.

Proof. Indeed, the conditions on the $\eta_{a, b}^{c}$ imply that the conditions in $\S 5.3$ hold for the elements $u_{a, b}^{c}$, so we get a homomorphism $\widetilde{E} \rightarrow J$. Since $J$ is torsion free, this descends to a homomorphism $E \rightarrow J$.

8.17. After modifying our choice of $\gamma_{A}$, we can arrange that $u=0$. The map $\gamma_{A}$ is also compatible with the $T$-action by construction.

Using Lemma 8.4, the map $\gamma_{A}$ defines a morphism

$$
K_{S} \rtimes P \longrightarrow H^{0}\left(\widetilde{X}_{A}, \mathscr{O}_{\widetilde{X}_{A}}\right),
$$

compatible with the $T$-action and whose image is contained in the $U$-invariants. Let $\mathcal{R}_{A}$ (respectively, $\mathcal{R}_{A}^{s t d}$ ) denote the coordinate ring of $\widetilde{X}_{A}$ (respectively, the base change of the cone over the standard family to $A$ ). Then it follows that there exists a unique $\widetilde{G}$-invariant map of $A$-modules

$$
\mathcal{R}_{A}^{s t d} \longrightarrow \mathcal{R}_{A}
$$

which on the highest weight vectors is given by $\gamma_{A}$. In fact, it follows from the construction that this is a map of $A$-algebras. Indeed, for any $a, b, c \in P$ with $(a, b) \succ c$ the $\mathcal{R}_{A, c}$-component of $\mathcal{R}_{A, a} \cdot \mathcal{R}_{A, b}$ is given by the image of $\eta_{a, b}^{c}$, viewed as an element of $K_{S}$ as in $\S 8.7$, under $\gamma_{A}$. This implies that the map (8.17.1) is a ring homomorphism. Since both algebras are flat over $A$, it follows that (8.17.1) is an isomorphism. From this Proposition 8.11 follows.

\section{Stable spherical log varieties}

Fix a subgroup $\Gamma \subset \widetilde{\Lambda}$ and an irreducible polarized spherical variety $\left(X_{0}, L_{0}\right)$ with weights in $\Gamma$.

Definition 9.1. Let $T$ be a scheme. A stable spherical log variety over $T$ with weights in $\Gamma$ adapted to $\left(X_{0}, L_{0}\right)$ is a collection of data

$$
\left(M_{T},\left(f, f^{b}\right):\left(X, M_{X}\right) \longrightarrow\left(T, M_{T}\right),\left(\rho, \rho^{b}\right), L, \underline{\eta}\right),
$$

where $\left(M_{T},\left(f, f^{b}\right):\left(X, M_{X}\right) \rightarrow\left(T, M_{T}\right),\left(\rho, \rho^{b}\right), L\right)$ satisfies conditions 8.1(i)-(vii) and $\underline{\eta}$ is a logarithmic enrichment, such that in every geometric fiber the isomorphism with the standard family occurring in $8.1(\mathrm{v})$ can be chosen to be compatible with the logarithmic enrichments and with starting variety the given $\left(X_{0}, L_{0}\right)$.

9.2. For two stable spherical log varieties with weights in $\Gamma$ and adapted to $\left(X_{0}, L_{0}\right)$ over a scheme $T$

$$
\left(M_{T}, f:\left(X, M_{X}\right) \longrightarrow\left(T, M_{T}\right), \rho, L_{X}, \underline{\eta}\right), \quad\left(M_{T}^{\prime}, f^{\prime}:\left(X^{\prime}, M_{X^{\prime}}\right) \longrightarrow\left(T, M_{T}^{\prime}\right), \rho^{\prime}, L_{X^{\prime}}, \underline{\eta}^{\prime}\right),
$$

an isomorphism between them is a triple $\left(\sigma,\left(g, g^{b}\right), r\right)$, where $\sigma: M_{T}^{\prime} \rightarrow M_{T}$ is an isomorphism of $\log$ structures on $T,\left(g, g^{b}\right):\left(X, M_{X}\right) \rightarrow\left(X^{\prime}, M_{X^{\prime}}\right)$ is an isomorphism of log schemes, and $r: g^{*} L_{X^{\prime}} \rightarrow L_{X}$ is an isomorphism of line bundles on $X$, such that the following conditions hold:

(i) The isomorphism $g$ is compatible with the $G$-action, and the pair $\left(g, g^{b}\right)$ is compatible with the $B$-action. 


\section{OLSSON}

(ii) The diagram

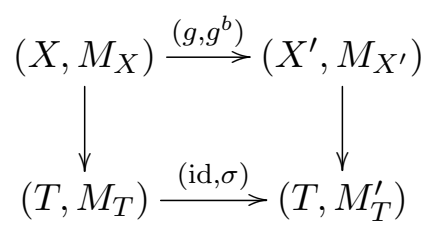

commutes.

(iii) The isomorphisms $\left(\sigma,\left(g, g^{b}\right)\right)$ are compatible with the logarithmic enrichments $\underline{\eta}$ and $\underline{\eta^{\prime}}$.

(iv) The isomorphism $r$ is compatible with the $G$-linearizations.

Let $\mathscr{S}_{\Gamma,\left(X_{0}, L_{0}\right)}^{\log }$ denote the fibered category over the category of $k$-schemes whose fiber over $T$ is the groupoid of stable spherical log varieties over $T$ with weights in $\Gamma$ and adapted to $\left(X_{0}, L_{0}\right)$.

9.3. We show in Theorem 9.6 below that $\mathscr{S}_{\Gamma,\left(X_{0}, L_{0}\right)}^{\log }$ is an algebraic stack. The key ingredient is understanding the isomorphisms between two objects of $\mathscr{S}_{\Gamma,\left(X_{0}, L_{0}\right)}^{\log }$.

So fix two objects

$$
\left(M_{T},\left(X, M_{X}\right), \rho, L_{X}, \underline{\eta}\right),\left(M_{T}^{\prime},\left(X^{\prime}, M_{X^{\prime}}\right), \rho^{\prime}, L_{X^{\prime}}, \underline{\eta^{\prime}}\right) \in \mathscr{S}_{\Gamma,\left(X_{0}, L_{0}\right)}^{\log }(T)
$$

over a scheme $T$. Let $I^{\text {log }}$ denote the functor on the category of $T$-schemes which to any $T^{\prime} / T$ associates the set of isomorphisms

$$
\left(M_{T},\left(X, M_{X}\right), \rho, L_{X}, \underline{\eta}\right)_{T^{\prime}} \longrightarrow\left(M_{T}^{\prime},\left(X^{\prime}, M_{X^{\prime}}\right), \rho^{\prime}, L_{X^{\prime}}, \underline{\eta^{\prime}}\right)_{T^{\prime}}
$$

in $\mathscr{S}_{\Gamma,\left(X_{0}, L_{0}\right)}^{\log }\left(T^{\prime}\right)$, where we write $\left(M_{T},\left(X, M_{X}\right), \rho, L_{X}, \underline{\eta}\right)_{T^{\prime}}$ etc. for the base change to $T^{\prime}$. Let $I$ denote the functor sending $T^{\prime} / T$ to the set of pairs $(a, b)$, where $a: X_{T^{\prime}} \rightarrow X_{T^{\prime}}^{\prime}$ is a $G$-equivariant isomorphism over $T^{\prime}$ and $b: a^{*} L_{X_{T^{\prime}}^{\prime}} \rightarrow L_{X_{T^{\prime}}}$ is an isomorphism of line bundles on $X_{T^{\prime}}$.

Lemma 9.4. The functor $I$ is representable by a $T$-scheme which is Zariski locally on $T$ quasiprojective over $T$.

Proof. We may work Zariski locally on $T$, and can therefore assume that $T$ is an affine scheme. Let $J$ be the functor sending $T^{\prime} / T$ to the set of $G$-equivariant isomorphisms $X_{T^{\prime}} \rightarrow X_{T^{\prime}}^{\prime}$. Then $J$ is a quasi-projective scheme represented by a locally closed subscheme of the Hilbert scheme of $X \times X^{\prime}$. In particular, $J$ is a quasi-projective scheme. Over $J$ there is a tautological isomorphism $a_{J}: X_{J} \rightarrow X_{J}^{\prime}$. The two invertible sheaves $L_{X}$ and $a_{J}^{*} L_{X^{\prime}}$ therefore define two morphisms $J \rightarrow$ Pic $_{X / T}$ to the relative Picard scheme of $X / T$. Let $H \subset J$ be the fiber product of the diagram

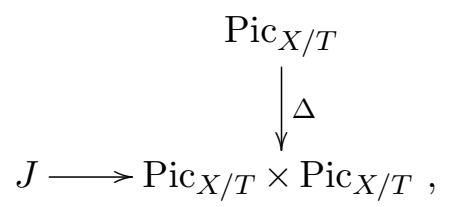

where the horizontal morphism is given by $\left[L_{X}\right] \times\left[a_{J}^{*} L_{X^{\prime}}\right]$. Then $H$ is again a quasi-projective scheme over $T$. Let $a_{H}$ denote the restriction of $a_{J}$ to $H$. Then the two invertible sheaves $L_{X}$ and $a_{H}^{*} L_{X^{\prime}}$ on $H$ are locally isomorphic for the faithfully flat of finite presentation topology. Let $M$ denote $L_{X} \otimes a_{H}^{*} L_{X^{\prime}}^{-1}$. Then $f_{*} M$ is a locally free sheaf of rank 1 on $H$. The functor $I$ is then isomorphic to the functor over $H$ of $G$-equivariant trivializations of $M$, which is quasi-projective over $T$. 


\section{MOduli OF SPHERICAL VARIETIES AND LOG GEOMETRY}

Proposition 9.5. The forgetful map $i: I^{\log } \rightarrow I$ is an isomorphism. In particular, $I^{\log }$ is a scheme which is Zariski locally on $T$ quasi-projective over $T$.

Proof. Let $T^{\prime}$ be a $T$-scheme, and let

$$
\left(a: X_{T^{\prime}} \longrightarrow X_{T^{\prime}}^{\prime}, b: a^{*} L_{X_{T^{\prime}}^{\prime}} \longrightarrow L_{X_{T^{\prime}}}\right)
$$

be a $G$-equivariant isomorphism. We have to show that this extends uniquely to an isomorphism in $\mathscr{S}_{\Gamma,\left(X_{0}, L_{0}\right)}^{\log }$. For this we may as well replace $T$ by $T^{\prime}$, so we may assume $T=T^{\prime}$.

Consider first the case when $T=\operatorname{Spec}(k)$ for $k$ an algebraically closed field. In this case we have pavings $S$ and $S^{\prime}$ of $Q$ such that the family

$$
\left(M_{k},\left(X, M_{X}\right), \rho, L_{X}\right) \quad\left(\text { respectively, }\left(M_{k}^{\prime},\left(X^{\prime}, M_{X^{\prime}}\right), \rho^{\prime}, L_{X^{\prime}}\right)\right)
$$

is induced by base change along the vertex map $k\left[K_{S}\right] \rightarrow k$ (respectively, $k\left[K_{S^{\prime}}\right] \rightarrow k$ ) from the standard family. We claim $S=S^{\prime}$.

Let $R$ (respectively, $R^{\prime}$ ) denote the affine cone of $\left(X, L_{X}\right)$ (respectively, $\left(X^{\prime}, L_{X^{\prime}}\right)$ ), so we get a $\widetilde{G}$-equivariant isomorphism $\sigma: R \rightarrow R^{\prime}$. This induces an isomorphism $\sigma_{\lambda}: R_{\lambda} \rightarrow R_{\lambda}^{\prime}$ for all $\lambda$. Now by the construction of the standard family, we have isomorphisms $R_{\lambda} \simeq V(\lambda)$ and $R_{\lambda}^{\prime} \simeq V(\lambda)$, and the map $\sigma_{\lambda}$ is identified with multiplication by an element $u_{\lambda} \in k^{*}$. Let $v_{\lambda} \in V(\lambda)$ be a highest weight vector as before. The paving $S$ (respectively, $S^{\prime}$ ) is characterized by the condition that $v_{\lambda} \cdot v_{\mu}=0$ in $R$ (respectively, $R^{\prime}$ ) if and only if $\lambda$ and $\mu$ lie in a common element of $S$. It follows that $S=S^{\prime}$.

Therefore our two objects are isomorphic, and the proof in the case when $T=\operatorname{Spec}(k)$ is reduced to showing that an automorphism $(a, b)$ of the underlying polarized $G$-scheme $\left(X, L_{X}\right)$ extends uniquely to an automorphism in $\mathscr{S}_{\Gamma,\left(X_{0}, L_{0}\right)}^{\log }(k)$. Let $H_{S} \subset K_{S}$ be as in Remark 6.4 and let $N \subset M_{T}$ be the sub-log structure defined by $H_{S}$. By the same argument as in the previous paragraph, the map $H_{S} \rightarrow k$ is induced by a map $H_{S}^{\text {sat }} \rightarrow k$ sending all nonunits to 0 . Let $M_{R}$ denote the $\log$ structure on the $\operatorname{cone} \operatorname{Spec}(R)$ induced by the map $K_{S} \rtimes P \rightarrow R^{U}$, and let $N_{R} \subset M_{R}$ be the sub-log structure obtained from the submonoid $P \rtimes H_{S} \subset K_{S} \rtimes P$. We then have a cartesian diagram of $\log$ schemes with $\widetilde{B}$-action

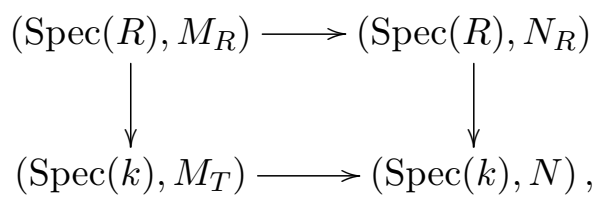

where the horizontal morphisms are the identity maps on the underlying schemes. The log structure $N_{R}$ is by construction obtained from a log structure $N_{R^{U}}$ on $\operatorname{Spec}\left(R^{U}\right)$, and the morphism

$$
\left(\operatorname{Spec}\left(R^{U}\right), N_{R^{U}}\right) \longrightarrow(\operatorname{Spec}(k), N)
$$

is a standard family in the sense of [Ols08a, 3.2.1]. By [Ols08a, Proposition 3.2.2] the pair $(a, b)$ therefore is induced by a unique $\widetilde{B}$-equivariant automorphism of $\left(N,\left(\operatorname{Spec}(R), N_{R}\right)\right)$. Let

$$
\left(\sigma^{\prime}: N \longrightarrow N,\left(\tilde{a}, \tilde{a}^{b}\right):\left(\operatorname{Spec}(R), N_{R}\right) \longrightarrow\left(\operatorname{Spec}(R), N_{R}\right)\right)
$$

denote this automorphism. It follows from the proof of [Ols08a, Proposition 3.2.2] that the induced map $\bar{\sigma}^{\prime}: \bar{N} \rightarrow \bar{N}$ is the identity, and therefore $\sigma$ is induced by a homomorphism $\rho^{\prime}: H_{S}^{\mathrm{gp}} \rightarrow$ $k^{*}$. Since $H_{S}^{\mathrm{gp}}$ is included into $K_{S}^{\mathrm{gp}}$, we can extend this to a homomorphism $\rho: K_{S}^{\mathrm{gp}} \rightarrow k^{*}$ to get an automorphism $\sigma: M_{T} \rightarrow M_{T}$ extending $\sigma$. Since the square (9.5.1) is cartesian, we therefore 


\section{OLSSON}

get an automorphism

$$
\left(\sigma: M_{T} \longrightarrow M_{T},\left(\tilde{a}, \tilde{a}^{b}\right):\left(\operatorname{Spec}(R), M_{R}\right) \longrightarrow\left(\operatorname{Spec}(R), M_{R}\right)\right)
$$

inducing the pair $(a, b)$, where we abusively also write $\left(\tilde{a}, \tilde{a}^{b}\right)$ for the automorphism of log schemes obtained from the automorphism in (9.5.2) by base change to $\left(\operatorname{Spec}(k), M_{T}\right)$. Note that while the data $\left(\sigma^{\prime}, \tilde{a}, \tilde{a}^{b}\right)$ is uniquely determined by $(a, b)$, this is not the case for $\sigma$. But we claim that there exists a unique choice of $\rho$ extending $\rho^{\prime}$ such that $\left(\sigma, \tilde{a}, \tilde{a}^{b}\right)$ is compatible with the logarithmic enrichments. Indeed, choose any extension $\rho$ as above, and for $c \in P$ let $\alpha_{c}: L_{c} \rightarrow L_{c}$ be the induced isomorphism. The failure of $\left(\sigma, \tilde{a}, \tilde{a}^{b}\right)$ to be compatible with the logarithmic enrichment is measured by the squares

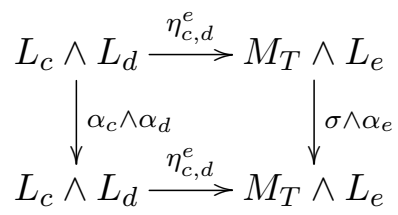

defined for each $c, d, e \in P$ with $(c, d) \succ e$. The map $\alpha_{c}$ is given by a unit $\gamma_{c} \in k^{*}$ and the condition that (9.5.3) commute amounts to having the equality

$$
\gamma_{e} \cdot \rho\left(\eta_{c, d}^{e}\right)=\gamma_{c} \cdot \gamma_{d}
$$

for all $(c, d) \succ e$. Now observe that the $\gamma_{c}$ define a homomorphism

$$
\lambda: \underset{\omega \in S}{\lim _{\omega}} P_{\omega}^{\mathrm{gp}} \longrightarrow k^{*},
$$

so, modifying the choice of $\rho$ by the homomorphism

$$
K_{S}^{\mathrm{gp}} \longrightarrow k^{*}, \quad x \longmapsto \rho(x)^{-1} \cdot \lambda(x),
$$

we get the desired extension of $\rho^{\prime}$. From this it is also clear that the choice of $\rho$ is unique subject to the condition that it be compatible with the enrichments.

Next, we consider the case when $T=\operatorname{Spec}\left(A^{\prime}\right)$ is an artinian local ring with residue field $k$, and $J \subset A^{\prime}$ is an ideal annihilated by the maximal ideal of $A^{\prime}$ and such that the result holds over $A:=A^{\prime} / J$. Let $\left(\sigma,\left(g, g^{b}\right), r\right) \in I^{\log }(A)$ be an isomorphism over $A$. We show how to lift this to an isomorphism $\left(\sigma^{\prime},\left(g^{\prime}, g^{\prime b}\right), r^{\prime}\right) \in I^{\log }\left(A^{\prime}\right)$ inducing the given isomorphism $(a, b) \in I\left(A^{\prime}\right)$. By descent theory (using the uniqueness) we may assume that $k$ is algebraically closed.

By Proposition 8.11 and using the case of an algebraically closed field, there exist maps $\beta, \beta^{\prime}: K_{S} \rightarrow A^{\prime}$ such that the two objects (9.3.1) are obtained by base change along these maps from the standard family. Furthermore, the isomorphism $\sigma$ over $A$ is induced by a homomorphism $u: K_{S}^{\mathrm{gp}} \rightarrow A^{*}$ such that for every $m \in K_{S}$ we have $\bar{\beta}^{\prime}(m)=u(m) \cdot \bar{\beta}(m)$, where $\bar{\beta}, \bar{\beta}^{\prime}: K_{S} \rightarrow A$ are the maps induced by $\beta$ and $\beta^{\prime}$.

Let $R_{A^{\prime}}$ (respectively, $R_{A^{\prime}}^{\prime}$ ) denote the coordinate ring of the cone of $\left(X, L_{X}\right)$ (respectively, $\left(X^{\prime}, L_{X^{\prime}}\right)$ ), and let $\tilde{a}: R_{A^{\prime}} \rightarrow R_{A^{\prime}}^{\prime}$ be the isomorphism induced by $(a, b)$. For $c \in P$, let $R_{A^{\prime}, c}$ (respectively, $R_{A^{\prime}, c}^{\prime}$ ) denote the corresponding subrepresentation of $R_{A^{\prime}}$ (respectively, $R_{A^{\prime}}^{\prime}$ ). The presentation of the objects (9.3.1) as induced by base change from the standard family defines trivializations of each $R_{A^{\prime}, c}$ and $R_{A^{\prime}, c}^{\prime}$, and using these trivializations the map $\tilde{a}$ is induced by a map

$$
\gamma: \lim _{\omega \in S} P_{\omega}^{\mathrm{gp}} \longrightarrow A^{*}
$$

Let $u^{\prime}: K_{S}^{\mathrm{gp}} \rightarrow A^{\prime *}$ be the restriction of this map to $K_{S}^{\mathrm{gp}}$. Then $u^{\prime}$ restricts to $u$ over $A$. By 


\section{MODULI OF SPHERICAL VARIETIES AND LOG GEOMETRY}

construction for any $c, d, e \in P$ with $(c, d) \succ e$ the diagram

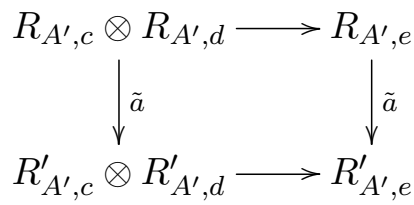

commutes, where the horizontal morphisms are induced by the multiplication map and the vertical morphisms are induced by $\tilde{a}$. From this it follows that for every $m \in K_{S}$ we have $\beta^{\prime}(m)=u^{\prime}(m) \cdot \beta(m)$, and therefore $u^{\prime}$ defines a lifting $M_{A^{\prime}} \rightarrow M_{A^{\prime}}$ of $\sigma$. This also shows that the morphism $\tilde{a}$ extends to an element of $I^{\log }\left(A^{\prime}\right)$. The uniqueness of this extension also follows from the preceding discussion.

Having treated the case of an artinian local ring, we get the extension of $(a, b)$ to an element of $I^{\log }$ in the case of a complete local ring by passing to the limit as in [Ols08a, proof of Lemma 3.7.6].

From this we deduce that if $T$ is a $k$-scheme, then the map

$$
I^{\log }(T) \longrightarrow I(T)
$$

is bijective, as follows. Both $I$ and $I^{\log }$ are sheaves for the étale topology on the category of $k$-schemes, so to prove that (9.5.4) is bijective we may replace $T$ by an étale cover, which reduces the proof to the case when $T$ is affine. In this case write $T=\lim _{j \in J} T_{j}$ as a projective limit of affine $k$-schemes $T_{j}$ of finite type. By a standard limit argument the objects (9.3.1) are defined over some $T_{j}$ and any element of $I(T)$ is induced by an element of $I\left(T_{j}\right)$ for some $j$. From this it follows that it suffices to prove the bijectivity of (9.5.4) in the case when $T$ is an affine scheme of finite type over $k$. In this case the Artin approximation theorem and the case when $T$ is a complete noetherian local ring imply that for any $(a, b) \in I(T)$ there exists an étale neighborhood of any point of $T$ over which $(a, b)$ lifts uniquely to an element of $I^{\log }(T)$. Using again that $I^{\log }$ and $I$ are sheaves for the étale topology, this implies that $I^{\log }(T) \rightarrow I(T)$ is a bijection in general.

This completes the proof of Proposition 9.5.

THEOREM 9.6. The fibered category $\mathscr{S}_{\Gamma,\left(X_{0}, L_{0}\right)}^{\log }$ is a quasi-separated algebraic stack.

Proof. We have just shown that the diagonal of $\mathscr{S}_{\Gamma,\left(X_{0}, L_{0}\right)}^{\log }$ is representable by separated schemes, so it remains to exhibit a smooth cover. Such a cover is provided by the standard families for various pavings $S$, by Proposition 8.11 .

\section{Global moduli}

10.1. Fix a subgroup $\Gamma \subset \widetilde{\Lambda}$ and a finite-dimensional $G$-representation $V$. Following $[\mathrm{AB} 06,4.1]$ we define $\mathscr{M}_{\Gamma, \mathbb{P} V}$ to be the stack whose fiber over a $k$-scheme $T$ is the groupoid of triples $(X, L, \epsilon)$, where $(X, L)$ is a stable polarized spherical variety over $T$ with weights in $\Gamma$ and $\epsilon: V \rightarrow \Gamma(X, L)$ is a morphism of $G$-representations which induces a finite map $\pi: X \rightarrow \mathbb{P} V_{T}$ such that the map $\pi^{*} \mathscr{O}_{\mathbb{P} V}(1) \rightarrow L$ (defined by $\epsilon$ ) is an isomorphism.

By $\left[\right.$ AB06, Remark 4.1.2(3)] the stack $\mathscr{M}_{\Gamma, \mathbb{P} V}$ is a proper algebraic stack with finite diagonal over $k$.

10.2. There are certain distinguished (or main) components of $\mathscr{M}_{\Gamma, \mathbb{P} V}$ defined as follows.

First of all, we have an open substack $\mathscr{M}_{\Gamma, \mathbb{P} V}^{\circ} \subset \mathscr{M}_{\Gamma, \mathbb{P} V}$ classifying triples $(X, L, \epsilon)$ with $X$ 


\section{OLSSON}

irreducible. For any such triple, the weight set $\widetilde{\Lambda}^{+}(X, L) \subset \Gamma$ is a submonoid which is locally constant in families. For each submonoid $P \subset \Gamma$ we therefore have an open and closed substack $\mathscr{M}_{\Gamma, \mathbb{P} V, P}^{\circ} \subset \mathscr{M}_{\Gamma, \mathbb{P} V}^{\circ}$ classifying triples $(X, L, \epsilon)$ with weight monoid $P$.

For any scheme $T$ and object $(X, L, \epsilon) \in \mathscr{M}_{\Gamma, \mathbb{P} V, P}^{\circ}(T)$, we can consider the relative cone $\widetilde{X}$ and the quotient $\widetilde{X} / / U$. This quotient is a family of toric varieties with torus $D\left(P^{\text {gp }}\right)$ which étale locally on $T$ is isomorphic to $Y_{P, T}:=\operatorname{Spec}(k[P]) \times_{\operatorname{Spec}(k)} T$.

As in [AB05, Definition 1.10], let $M_{Y_{P}}$ denote the scheme classifying affine $\widetilde{G}$-varieties of type $Y_{P}$. So for a scheme $T$ an element of $M_{Y_{P}}(T)$ is given by a family of affine $\widetilde{G}$-schemes $\pi: Z \rightarrow T$ and an isomorphism $\varphi: Z / / U \simeq Y_{P, T}$.

Note that the automorphism group scheme of $Y_{P, T}$ (as a toric variety) is $D\left(P^{\mathrm{gp}}\right)$, and therefore there is an action of $D\left(P^{\mathrm{gp}}\right)$ on $M_{Y_{P}}$ obtained by modifying the choice of isomorphism $\varphi$. By [AB05, Corollary 3.4] this action has finitely many orbits.

Now let $\left(X_{0}, L_{0}\right)$ be an irreducible polarized spherical variety over $k$ with weight monoid $P$. Choosing an isomorphism $\widetilde{X}_{0}^{U} \simeq Y_{P}$, we get a point of $M_{Y_{P}}$, and the corresponding $D\left(P^{\text {gp }}\right)$-orbit $O_{\left(X_{0}, L_{0}\right)}$ is independent of the choice of isomorphism. We say that $\left(X_{0}, L_{0}\right)$ is generic if the orbit $O_{\left(X_{0}, L_{0}\right)}$ is open in $M_{Y_{P}}$.

10.3. Let $\left(X_{0}, L_{0}\right)$ be a fixed irreducible polarized spherical variety over $k$ with weight monoid $P \subset \Gamma$ and assume that $\left(X_{0}, L_{0}\right)$ is generic. Define $\mathscr{M}_{\Gamma, \mathbb{P} V,\left(X_{0}, L_{0}\right)}^{\log }$ to be the fibered category over the category of $k$-schemes whose fiber over a scheme $T$ is the groupoid of data

$$
\left(\left(M_{T},\left(f, f^{b}\right):\left(X, M_{X}\right) \longrightarrow\left(T, M_{T}\right),\left(\rho, \rho^{b}\right), L, \underline{\eta}\right), \epsilon\right),
$$

where

$$
\left(M_{T},\left(f, f^{b}\right):\left(X, M_{X}\right) \longrightarrow\left(T, M_{T}\right),\left(\rho, \rho^{b}\right), L, \underline{\eta}\right) \in \mathscr{S}_{\Gamma,\left(X_{0}, L_{0}\right)}^{\log }(T)
$$

and $\epsilon: V \rightarrow \Gamma(X, L)$ is a morphism of $G$-representations such that the collection $(X, L, \epsilon)$ is an object of $\mathscr{M}_{\Gamma, \mathbb{P} V}(T)$.

There is a forgetful map

$$
\tau: \mathscr{M}_{\Gamma, \mathbb{P} V,\left(X_{0}, L_{0}\right)}^{\log } \longrightarrow \mathscr{M}_{\Gamma, \mathbb{P} V} .
$$

Lemma 10.4. Let $A$ be a discrete valuation ring over $k$ with field of fractions $K$ and residue field $\kappa$, and let $(X, L, \epsilon) \in \mathscr{M}_{\Gamma, \mathbb{P} V}(A)$. Suppose that the closed fiber $\left(X_{\kappa}, L_{\kappa}\right)$ of $(X, L)$ is irreducible with weight monoid $P$ and generic. Then there exists a polarized spherical variety $\left(X_{0}, L_{0}\right)$ over $k$ with weight monoid $P$ which is generic and such that, after possibly replacing $A$ by a finite extension, we have $(X, L) \simeq\left(X_{0}, L_{0}\right) \otimes_{k} A$.

Proof. Let $\mathcal{R}$ be the cone of $(X, L)$, which is a stable affine spherical variety over $A$. By [AB06, Proposition 3.1.4], after replacing $A$ by a finite extension, we may assume that $\mathcal{R}$ is standard in the terminology of loc. cit. This means that there exists a polarized spherical variety $\left(X_{0}, L_{0}\right)$ over $k$ such that if $R_{0}$ denotes the corresponding cone, then we have an inclusion $\mathcal{R} \hookrightarrow R_{0} \otimes_{k} K$ of algebras with $\widetilde{G}$-action over $A$ which induces an isomorphism over $K$. Let $\pi \in A$ be a uniformizer and let $P \subset \Gamma$ be the monoid of weights occurring in $R_{0}$. Then for each $\lambda \in P$ the image of $\mathcal{R}_{\lambda}$ in $R_{0, \lambda} \otimes_{k} K$ is equal to $\pi^{h(\lambda)} R_{0} \otimes_{k} A$ for some function $h: P \rightarrow \mathbb{Z}$ admissible with respect to $\left(X_{0}, L_{0}\right)$. Now observe that since the closed fiber of $(X, L)$ is irreducible, the function $h$ is in fact linear. It follows that the $T$-orbit $O_{\left(X_{\kappa}, L_{\kappa}\right)}$ is in the closure of the orbit $O_{\left(X_{K}, L_{K}\right)}$. Since $\left(X_{\kappa}, L_{\kappa}\right)$ was assumed generic, this implies $O_{\left(X_{\kappa}, L_{\kappa}\right)}=O_{\left(X_{K}, L_{K}\right)}$, which in turn implies the result. 


\section{MODULI OF SPHERICAL VARIETIES AND LOG GEOMETRY}

Lemma 10.5. There exists a unique open substack $\mathscr{M}_{\Gamma, \mathbb{P} V,\left(X_{0}, L_{0}\right)}^{\circ} \subset \mathscr{M}_{\Gamma, \mathbb{P} V}$ such that a geometric point $\bar{x} \rightarrow \mathscr{M}_{\Gamma, \mathbb{P} V}$ corresponding to an object $(X, L, \epsilon)$ is in $\mathscr{M}_{\Gamma, \mathbb{P} V,\left(X_{0}, L_{0}\right)}^{\circ}$ if and only if $(X, L) \simeq$ $\left(X_{0}, L_{0}\right) \otimes_{k} k(\bar{x})$.

Proof. The uniqueness is clear.

For the existence, let $(\mathscr{X}, \mathscr{L})$ be the universal polarized scheme over $\mathscr{M}_{\Gamma, \mathbb{P} V}$. By Lemma 9.4 the functor over $\mathscr{M}_{\Gamma, \mathbb{P} V}$ classifying isomorphisms between $(\mathscr{X}, \mathscr{L})$ and $\left(X_{0}, L_{0}\right)$ is a relative scheme $\mathscr{I} \rightarrow \mathscr{M}_{\Gamma, \mathbb{P} V}$, locally quasi-projective over $\mathscr{M}_{\Gamma, \mathbb{P} V}$. Let $V \rightarrow \mathscr{M}_{\Gamma, \mathbb{P} V}$ be a smooth morphism with $V$ an affine scheme and let $\mathscr{I}_{V} \rightarrow V$ be the base change of $\mathscr{I}$. Then the image $V^{\prime} \subset V$ of $\mathscr{I}_{V}$ in $V$ is a constructible subset and we need to show that it is open. For this it suffices to show that if $A$ is a discrete valuation ring and $\operatorname{Spec}(A) \rightarrow V$ is a morphism sending the closed point of $\operatorname{Spec}(A)$ to $V^{\prime}$, then the generic point of $\operatorname{Spec}(A)$ also maps to $V^{\prime}$. Equivalently, we need to show that if $A$ is a discrete valuation ring with field of fractions $K$ and algebraically closed residue field $\kappa$, and if $(X, L, \epsilon)$ is an object of $\mathscr{M}_{\Gamma, \mathbb{P} V}$ over $A$ such that $\left(X_{\kappa}, L_{\kappa}\right) \simeq\left(X_{0}, L_{0}\right) \otimes_{k} \kappa$, then $\left(X_{K}, L_{K}\right) \simeq\left(X_{0}, L_{0}\right) \otimes_{k} K$, after possibly replacing $A$ by an extension. This follows from Lemma 10.4 .

Let $\mathscr{M}_{\Gamma, \mathbb{P} V,\left(X_{0}, L_{0}\right)} \subset \mathscr{M}_{\Gamma, \mathbb{P} V}$ be the scheme-theoretic closure of $\mathscr{M}_{\Gamma, \mathbb{P} V,\left(X_{0}, L_{0}\right)}^{\circ}$.

Theorem 10.6. The map $\tau$ in (10.3.1) factors through a finite universal homeomorphism

$$
\tau^{\prime}: \mathscr{M}_{\Gamma, \mathbb{P} V,\left(X_{0}, L_{0}\right)}^{\log } \longrightarrow \mathscr{M}_{\Gamma, \mathbb{P} V,\left(X_{0}, L_{0}\right)} \cdot
$$

In particular, $\mathscr{M}_{\Gamma, \mathbb{P} V,\left(X_{0}, L_{0}\right)}^{\log }$ is a proper algebraic stack with finite diagonal.

Proof. For an object $\left(M_{T},\left(f, f^{b}\right):\left(X, M_{X}\right) \rightarrow\left(T, M_{T}\right),\left(\rho, \rho^{b}\right), L, \underline{\eta}\right)$ of $\mathscr{S}_{\Gamma,\left(X_{0}, L_{0}\right)}^{\log }$ over a scheme $T$ and a morphism $\epsilon: V \rightarrow \Gamma(X, L)$, the condition that $(X, L, \epsilon)$ define an object of $\mathscr{M}_{\Gamma, \mathbb{P} V}$ is an open condition on $T$. If $(\mathcal{X}, \mathcal{L})$ is the universal polarized scheme over $\mathscr{S}_{\Gamma,\left(X_{0}, L_{0}\right)}^{\log }$ and $f: \mathcal{X} \rightarrow \mathscr{S}_{\Gamma,\left(X_{0}, L_{0}\right)}^{\log }$ is the structure morphism, then $f_{*} \mathcal{L}$ is a locally free sheaf on $\mathscr{S}_{\Gamma,\left(X_{0}, L_{0}\right)}^{\log }$ whose formation commutes with arbitrary base change (by [AB06, Proposition 2.2.3]), and $\mathscr{M}_{\Gamma, \mathbb{P} V,\left(X_{0}, L_{0}\right)}^{\log }$ is realized as an open substack of the total space of $V^{\vee} \otimes f_{*} \mathcal{L}$. In particular, $\mathscr{M}_{\Gamma, \mathbb{P} V,\left(X_{0}, L_{0}\right)}^{\log }$ is an algebraic stack by Theorem 9.6. Furthermore, the map $\tau$ is fully faithful by Proposition 9.5. Therefore the diagonal of $\mathscr{M}_{\Gamma, \mathbb{P} V,\left(X_{0}, L_{0}\right)}^{\log }$ is finite.

The map $\tau^{\prime}$ is also proper. Indeed, by [DM69, paragraph preceding Proposition 4.20] applied to the base change of $\tau^{\prime}$ to a smooth cover of $\mathscr{M}_{\Gamma, \mathbb{P} V,\left(X_{0}, L_{0}\right)}$, to verify this it suffices to show that for any discrete valuation ring $A$ and morphism $g: \operatorname{Spec}(A) \rightarrow \mathscr{M}_{\Gamma, \mathbb{P} V,\left(X_{0}, L_{0}\right)}$ sending the generic point of $\operatorname{Spec}(A)$ to $\mathscr{M}_{\Gamma, \mathbb{P} V,\left(X_{0}, L_{0}\right)}^{\circ}$ the morphism $g$ lifts to $\mathscr{M}_{\Gamma, \mathbb{P} V,\left(X_{0}, L_{0}\right)}^{\log }$. This follows from $[\mathrm{AB} 06$, Proposition 3.1.4].

Thus our morphism $\tau^{\prime}$ is a finite morphism of algebraic stacks such that for any algebraically closed field $\kappa$ the induced map

$$
\mathscr{M}_{\Gamma, \mathbb{P} V,\left(X_{0}, L_{0}\right)}^{\log }(\kappa) \longrightarrow \mathscr{M}_{\Gamma, \mathbb{P} V,\left(X_{0}, L_{0}\right)}(\kappa)
$$

is an equivalence of categories (again using Proposition 9.5). This implies that $\tau^{\prime}$ is a universal homeomorphism. 


\section{OLSSON}

\section{ACKNOWLEDGEMENTS}

We thank Ravi Vakil and the referee for helpful comments.

\section{REFERENCES}

AB05 V. Alexeev and M. Brion, Moduli of affine schemes with reductive group action, J. Algebraic Geom. 14 (2005), no. 1, 83-117; http://dx.doi.org/10.1090/S1056-3911-04-00377-7.

AB06 - Stable spherical varieties and their moduli, IMRP Int. Math. Res. Pap. 2006 (2006), Art. ID 46293; http://dx.doi.org/10.1155/IMRP/2006/46293.

DM69 P. Deligne and D. Mumford, The irreducibility of the space of curves of given genus, Publ. Math. Inst. Hautes Études Sci. 36 (1969), 75-109; http://dx.doi.org/10.1007/BF02684599.

Kat94 K. Kato, Toric singularities, Amer. J. Math. 116 (1994), no. 5, 1073-1099; http://dx.doi.org/ 10.2307/2374941.

Los09 I. V. Losev, Proof of the Knop conjecture, Ann. Inst. Fourier (Grenoble) 59 (2009), no. 3, 11051134, http://dx.doi.org/10.5802/aif.2459.

Ogu14 A. Ogus, Lectures on logarithmic algebraic geometry, book in preparation, version of August 13, 2014, available at https://math.berkeley.edu/ ogus/.

Ols08a M. Olsson, Compactifying moduli spaces for abelian varieties, Lecture Notes in Math., vol. 1958 (Springer-Verlag, Berlin, 2008); http://dx.doi.org/10.1007/978-3-540-70519-2.

Ols08b_ Logarithmic interpretation of the main component in toric Hilbert schemes, in Curves and Abelian Varieties, Contemp. Math., vol. 465 (Amer. Math. Soc., Providence, RI, 2008), 231-252.

Martin Olsson molsson@math.berkeley.edu

University of California, Berkeley, 970 Evans Hall, Berkeley, CA 94720-3840, USA 